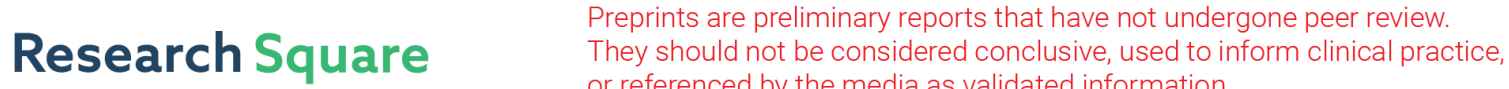 or referenced by the media as validated information. \\ Thermal Error Prediction of Ball Screws Based on PSO-LSTM
}

Xiangsheng Gao ( $\sim$ gaoxsh@bjut.edu.cn )

Beijing University of Technology https://orcid.org/0000-0001-5947-5826

Yueyang Guo

Dzonu Ambrose Hanson

Zhihao Liu

Min Wang

Tao Zan

\section{Research Article}

Keywords: Thermal error, Ball screw, Modeling, PSO-LSTM, Data-driven

Posted Date: March 23rd, 2021

DOI: https://doi.org/10.21203/rs.3.rs-328258/v1

License: (a) This work is licensed under a Creative Commons Attribution 4.0 International License. Read Full License 


\title{
Thermal error prediction of ball screws based on PSO-LSTM
}

\author{
Xiangsheng Gao*, Yueyang Guo, Dzonu Ambrose Hanson, Zhihao Liu, \\ Min Wang, Tao Zan
}

Beijing Key Laboratory of Advanced Manufacturing Technology, Faculty of Materials and Manufacturing, Beijing University of Technology, Beijing 100124, China.

*Corresponding author: Xiangsheng Gao, gaoxsh@bjut.edu.cn; Tel.:

+86-10-6739-2137/+86-130-3106-5766

Present address: Key Laboratory of Advanced Manufacturing Technology, Beijing University of

Technology, No. 100 Pingleyuan, Chaoyang District, Beijing, China (Mail code in China:

100124).

\begin{abstract}
:
Thermal error of ball screws seriously affects the machining precision of CNC machine tools especially in high speed and precision machining. Compensation technology is one of the most effective methods to address the thermal issue, and the effect of compensation depends on the accuracy and robustness of the thermal error model. Traditional modeling approaches have major challenges in time-series thermal error prediction. In this paper, a novel thermal error model based on Long Short-Term Memory (LSTM) neural network and Particle Swarm Optimization (PSO) algorithm is proposed. A data-driven model based on LSTM neural network is established according to the time-series collected data. The hyperparameters of LSTM neural network are optimized by PSO and then a PSO-LSTM model is established to precisely predict the thermal error of ball screws. In order to verify the effectiveness and robustness of the proposed model, two thermal characteristic experiments based on step and random speed are conducted on a self-designed test bench. The results show that the PSO-LSTM model has higher accuracy compared with the RBF model and BP model with high robustness. The proposed method can be implemented to predict the thermal error of ball screws, and provide a foundation for thermal error compensation.
\end{abstract}

Keywords Thermal error $\cdot$ Ball screw $\cdot$ Modeling $\cdot$ PSO-LSTM $\cdot$ Data-driven 


\section{Introduction}

Ball screws, an important transmission component which converts the rotary motion into the linear motion, have been widely applied in $\mathrm{CNC}$ machine tools owing to its high efficiency, precision and stiffness [1]. The productive efficiency of the machine tool is determined by the speed and feed rate of the machine tool, and it shows an obvious high-speed trend in recent years. However, severe thermal issues will follow the high speed and feed rate. The temperature rise results in thermally induced error of ball screws, which seriously affects the machining precision of $\mathrm{CNC}$ machine tools especially in high speed and precision machining. It is reported that thermal error represents $40-70 \%$ of the total error of machine tools [1-2]. Generally, the heat generation of ball screws is mainly from the motor, nuts and bearings, which causes the thermal elastic deformation of the screw resulting in loss of position accuracy [3]. Moreover, with the increasing of manufacturing precision, it is extremely important to reduce the influence of thermal issue of ball screws on position accuracy. Therefore, it is essential to implement the study on the thermal characteristic mechanism and the accurate modeling.

Reduction and compensation of the thermal deformation are the two main technical measures to address the thermal issues of ball screws. In terms of the thermal errors reduction, air cooling system, liquid cooling system and the adaptive method based on advanced materials have been developed in the past few years. Xu et al. [4-5], and Shi et al. [6] discussed the placement of an air/liquid cooling system in a ball screw shaft to overcome thermal errors and achieve temperature equilibrium faster. A series of tests were carried out and the results showed the position accuracy would be significantly improved. Nevertheless, the systems become more complicated and cause the loss of stiffness. Gao et al. [7] proposed an adaptive method based on carbon fiber reinforced plastics (CFRP) to reduce thermal deformation, where the fiber is mounted in the hollow along with the axial direction of the screw. When the temperature rises, the CFRP will contract the screw and reduce the thermal deformation due to the negative thermal expansion coefficient. But, the structure of ball screws in the research is difficult to implement in engineering practice because of the oversized clamping structure in the two ends. Guo et al. [8] proposed a bio-inspired graphene-coated ball screws inspired by the Saharan silver ant to reduce the thermal deformation. The high thermal emissivity of graphene coating on the screw is employed to quickly transfer the heat and radiate heat to the environment to reduce the temperature rise and thermal deformation. However, the coating may peel off from the surface of ball screw resulting in failure of the method.

Thermal error compensation is a cost-effective method to solve this problem, which merely depends on the exact measurement and the accurate prediction of thermal error during machining. Exact modeling of thermal errors is crucial to errors compensation and leads to good effect in compensation. Therefore, many researches have been carried out to address thermal errors prediction by using various methodologies. In the last decades, the most widely-used algorithms in the thermal error modeling were the multivariate linear regression analysis. Yang et al. [9] proposed a thermal error model based on cerebellar model articulation controller (CMAC) neural network which can search for the nonlinear and interaction characteristics between the thermal errors and temperature field on the machine tools. However, the convergence rates and prediction accuracy are not suitable since parameters of the model are not optimized. Yang et al. [10] decreased the errors due to temperature measuring noise and the adverse effect of environmental 
changes, and reduced the number of sensors according to thermal expansion and thermal bending mode analysis, and established the multivariate linear regression model to compensate the thermal error on a CNC turning center. Nevertheless, a practical regression model with high accuracy and robustness is difficult to establish by the method. Zhao et al. [11] proposed a method for determining the convection heat transfer coefficient, and then the temperature field and thermal errors were dynamically simulated using the finite element analysis (FEA) method satisfying to replace the experiment results. Zhu et al. [12] developed a temperature sensor placement scheme and thermal error modeling strategy based on simulations and experiments by using the finite element analysis, thermal mode concept and linear regression models. But the boundary conditions in actual working conditions haven't been mentioned in these studies. Some researchers focused on the mathematical model to reveal the error generation mechanism and established the analytical relationship between temperature rise of the temperature measurement points and thermal deformation for determining thermal error. However, the modeling method is less compatible since the variation of temperature rise is complicated leading to the analytic relationship variation with the working condition [13-14]. In recent years, with the development of computer technology and intelligent algorithm, gray theory, support vector machine, artificial neural network and some hybrid approaches were gradually employed by many researchers. Ramesh et al. [15-16] employed Bayesian networks to classify the thermal error in different parameter settings and adopted the support vector machine algorithm to determine the thermal error of each class. Wu et al. [17] established a GA-BPN-based thermal error model for online prediction of the thermal errors and developed a real-time compensation system to compensate for the thermal drift errors, while the performance of the model under complex conditions was not revealed. Zhang et al. [18] proposed a thermal error model by using the artificial neural networks and gray theory to enhance the robustness and the prediction accuracy of the model, but the weight coefficients seem difficult to be adjusted in real-time depending on the accuracy requirement and the actual model output. Wang et al. [19], Miao et al. [20-21] and Yang et al. [22] used a combination of fuzzy clustering analysis and advanced algorithm to establish the thermal error model. Abdulshahed et al. [23-24] used the ANFIS method and ANN with a back-propagation algorithm and ANN with a PSO algorithm combined with grey system theory to establish the thermal error prediction model. Liu et al. [25] established a thermal error model by using the ridge regression algorithm to inhibit the bad influence of collinearity on the thermal error predicted robustness. Dos Santos et al. [26] established physical models with data-driven models based on the ANN and the FEM simulation to predict the thermal error of a five-axis CNC machining center. Huang et al. [27] used a genetic algorithm (GA) to optimize initial weights and thresholds of BP network for training the thermal error sample data and modeling of the thermal error. Rojek et al. [28] used single-directional multi-layered neural networks with error back-propagation (MLP), radial basis function neural networks (RBF) and Kohonen networks to establish the predictive compensation model of ball screws. Li et al. [29] proposed a novel thermal error compensation model by using genetic algorithm to optimize wavelet neural network, which combines the advantages of adaptive global optimization searching ability of genetic algorithm and good time-frequency local characteristics of wavelet neural network. The robustness of thermal error model, variable searching and modeling time can be further optimized theoretically. Although these methods can enhance the predicted robustness of the model, the predicted accuracy reduced at the same time [23]. The thermal errors of machine tools can be predicted 
using these models by the mapping relationship between thermal error and temperature of selected sensitive points at certain moment. However, the robustness and accuracy of the model need to be improved since the thermal errors are not only related to temperature of selected sensitive points at some certain moment but also vary with historical temperature value. Therefore, it is necessary to establish an accurate real-time and historical mapping relationship between temperature fields and thermal errors. Long Short-Term Memory (LSTM) network as one of the modern deep learning models, has a strong ability for time series forecast in various fields, since it can dynamically learn new information while maintaining a persistent memory of historical information. Sagheer et al. [30] implemented time series forecasting of petroleum production based on LSTM recurrent networks optimized by genetic algorithm (GA), the empirical results showed that the LSTM model outperforms other standard approaches. Zhang et al. [31] employed the LSTM network to predict remaining useful life (RUL) of lithium-ion batteries. Zhang et al. [32] developed a LSTM model to predict water table depth in agricultural areas, evaluated and discussed the ability of the proposed model, and the results showed that the LSTM model had a satisfactory performance. Qin et al. [33] employed the LSTM model to predict gear remaining life. In a word, LSTM network has both the short-term correlation and long-term dependence characteristics. However, the research on thermal errors prediction of machine tools by using LSTM network is rarely reported, especially in the field of ball screws.

In this research, a novel thermal errors model of ball screws is proposed. Initially, a LSTM model is developed to forecast the time-series thermal errors of ball screws. In order to establish an accurate mapping relationship with time-varying between temperature fields and thermal errors, Particle swarm optimization algorithm (PSO) is employed to optimize the hyperparameters of LSTM network for improving the performance of the model. Furthermore, the effectiveness and robustness of the PSO-LSTM model are verified according to the collected experimental data. Finally, performance of the proposed model and traditional ones are compared. The rest of this paper is organized as follows. In Section 2, the modeling process and relevant theory of the proposed method are introduced. In Section 3, the thermal mode analysis and experiments are conducted, and the performance of proposed data-driven model is discussed and compared with other methods. Section 4 summarizes the main conclusions.

\section{Thermal error prediction of ball screws based on PSO-LSTM}

A deep learning model based on PSO-LSTM to predict thermally induced error of ball screws according to temperature and deformation data measured by temperature sensor and eddy current displacement sensor, respectively, is proposed in this research. And then the essential configuration of the deep learning model is optimized by Particle Swarm Optimization algorithm (PSO). The model is intended to quickly determine the thermal deformation and is used to compensate online thermal error in the working status. It is established and optimized by the Matlab deep learning toolbox and global optimization toolbox respectively.

\subsection{LSTM neural network}

Long short-term memory (LSTM) network, one of popular deep learning architectures in 
recent years, is developed specially to learn and handle long-term dependencies presented in sequential data such as temperature rise and thermal deformation of machine tools in the machining process. The exploding/vanishing gradient problem, which directly leads to the recurrent neural network (RNN) learning stopping or becoming too slow, can be solved by introducing a memory cell and three gating mechanism into the architecture of LSTM which modifies the RNN structure. Fig. 1 shows the framework of a LSTM neural network. The core of LSTM network is a cell memory state represented by the horizontal line through the cell which is similar to a conveyor belt running through the entire cell, but it only has few branches. It also includes three gates which called forget gate, input gate and output gate, to control and update cell status. Therefore, the network can ensure the entire information passes through the cell and update information to maintain its memory state with time. Based on the above characteristic, LSTM can not only address variable length time-series data and capture long-term dependencies, but also memorize historical information dynamically and learn new information while maintaining a persistent memory of historical information [34-37].

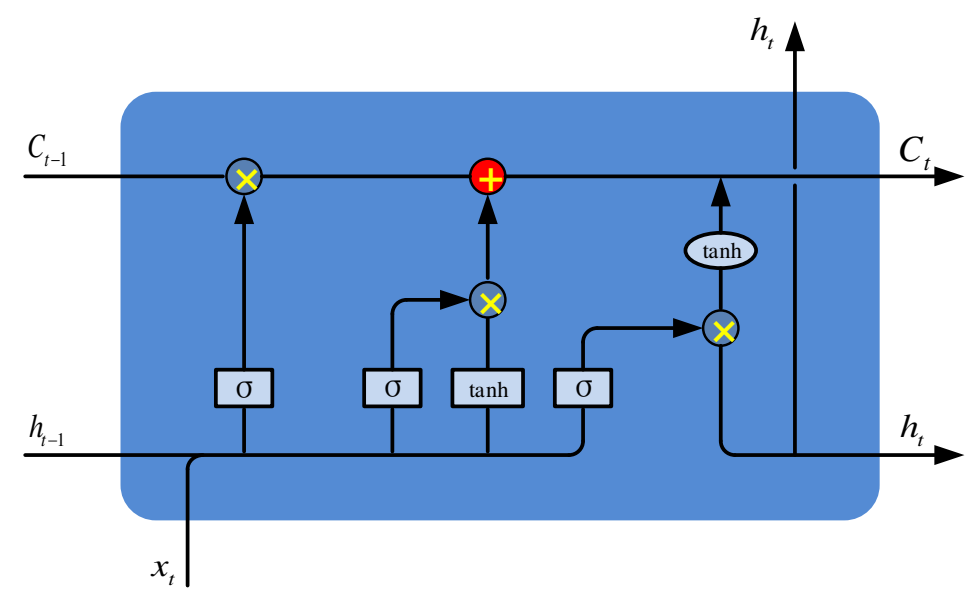

Fig. 1 Framework of the LSTM neural network

The first step of LSTM is removing unimportant information operated by a unit called forget gate, which can be derived as

$f_{t}=\sigma\left(W_{f} \cdot\left[h_{t-1}, x_{t}\right]+b_{f}\right)$

Where $f_{t}$ is on behalf of forget gate, $\sigma$ is the activation function, $W_{f}$ is the weight matric of forget gate, $h_{t-1}$ is the output at the previous time $t-1, x_{t}$ is the current input, and $b_{f}$ is a bias vector.

The next step is to select and add valuable information to the network through input gate, and to produce new cell information waiting to be selected. This process can be expressed as

$i_{t}=\sigma\left(W_{i} \cdot\left[h_{t-1}, x_{t}\right]+b_{i}\right)$

$C_{t}^{O D}=\tanh \left(W_{C} \cdot\left[h_{t-1}, x_{t}\right]+b_{C}\right)$

Where $i_{t}$ and $\mathcal{E}_{t}^{0}$ are the input gate and the intermediate value during the calculation, $W_{i}$ and $W_{C}$ are weight matrixes of input gate and internal state, $b_{i}$ and $b_{C}$ are biases of input gate and internal state.

Subsequently, new cell information is updated in the network through input gate and forget gate, which is formulated as

$C_{t}=f_{t} * C_{t-1}+i_{t} * C_{t}^{0}$

Where $C_{t}$ and $C_{t-1}$ are the cell state at the current time $t$ and the previous time $t-1$, 
respectively.

Finally, the outcome and cell state of LSTM is determined by updating and selecting the new cell information and the input, which is written as

$o_{t}=\sigma\left(W_{o} \cdot\left[h_{t-1}, x_{t}\right]+b_{o}\right)$

$h_{t}=o_{t} * \tanh \left(C_{t}\right)$

Where $o_{t}, \sigma, W_{o}$ and $b_{o}$ are the output gate, activation function, the weight matrix of output gate and the bias of output gate, respectively. $h_{t}$ is the output at the current time $t$.

The above equations reveal the internal calculation mechanism of LSTM where the network output of each time step is associated with previous input and cell state to predict future information by addressing variable length time-series data and capturing long-term dependencies. Therefore, LSTM can dynamically memorize historical information and learn new information while maintain a persistent memory of historical information.

\subsection{Thermal error modeling by LSTM}

Thermally induced error of ball screws can be processed by LSTM network as the temperature and deformation is time-series data increasing or dropping with time once the machine tool is working. Firstly, the essential configuration of LSTM network is designed based on the input data set and output data where the number of features is just in correspondence with temperature measurement point and thermal deformation respectively. The multi-layer LSTM and added full-connected layer are introduced into the network model. In the model training process, weighs and bias are updated by Adam optimizer and the root mean square error (RMSE) as fitness function is used to evaluate the performance of the LSTM. Additionally, the hyperparameters of LSTM is adjusted by optimization algorithm due to the ability to quickly find the best solution.

\subsection{Hyperparameter optimization algorithm}

In order to establish an ideal network with accurate performance, it is necessary to search optimal parameters of the model. Particle Swarm Optimization algorithm (PSO) is derived from the imitation of bird predation behavior. As one of the evolutionary calculation technologies, PSO can collaborate and share information between individuals in the group to find the optimal solution quickly so that it is suitable to be used for searching the optimal parameters. Therefore, PSO is applied in this study to optimize the network hyperparameters for better results. This algorithm is conducted by continuously searching and updating the personal best and global best of swarm while simultaneously the position and velocity of each particle are updating for the next optimization process. The search process of this algorithm is summarized in Fig. 2. 


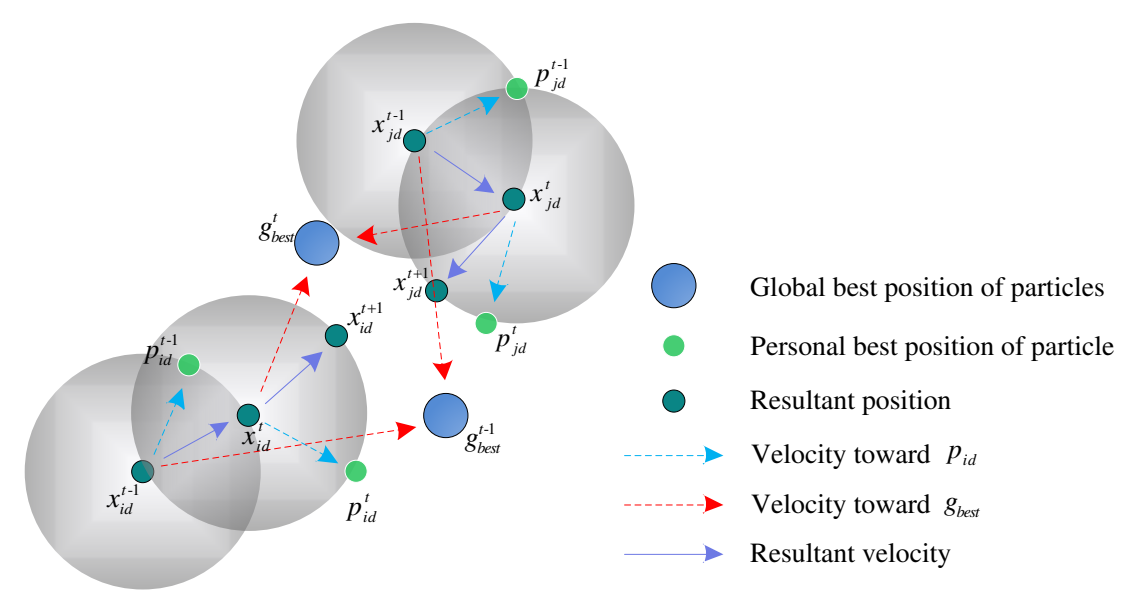

Fig. 2 Search process of particles

\subsection{Evaluation metrics of the model}

RMSE, MAE, MSE and MAPE are four metrics for evaluating the performance of the model. The smaller value of the metric indicates the better performance with the model, which are expressed as

$$
\begin{aligned}
& \text { RMSE }=\sqrt{\frac{1}{n} \sum_{i=1}^{n}\left(y_{i}-\$_{i}\right)^{2}} \\
& M A E=\frac{1}{n} \sum_{i=1}^{n}\left|y_{i}-\$_{i}\right| \\
& M S E=\frac{1}{n} \sum_{i=1}^{n}\left(y_{i}-\$_{i}\right)^{2} \\
& M A P E=\frac{100}{n} \sum_{i=1}^{n}\left|\frac{y_{i}-\$_{i}}{y_{i}}\right|
\end{aligned}
$$

Where $n$ is the number of samples, and $y_{i}$ and $\$_{i}$ are the actual and predictive values of $i$ -th sample, respectively.

\subsection{Thermal error prediction based on PSO-LSTM model}

Learning rate is the most crucial hyperparameters, followed by the network size [38]. In this paper, the hyperparameters of LSTM network, learning rate and unit number, were searched by PSO intelligent optimization algorithm by using the fitness function of root mean square error to search the optimal learning rate and unit number under the best fitness value. Simultaneously, the time window size of data set is designed by using PSO where the time window is introduced into the data set to improve the accuracy of the model. In this research, the relationship between thermal deformation and temperature rise of measured points is given by

$Y_{t}=f\left(Y_{t-1}, T_{t-1}, T_{t-2}, \cdots, T_{t-n}\right)$

Where $Y_{t}$ and $Y_{t-1}$ is on behalf of the predicted thermal error at the current time $t$ and the previous time $t-1, T_{t-1}, T_{t-2}, \cdots, T_{t-n}$ is the previous temperatures and the number $\mathrm{n}$ is the time window size. 
The time-series data of temperature and thermal deformation in axial direction are measured by temperature sensor and eddy current displacement sensor respectively. And then these data are imported into the LSTM network to train the data-driven LSTM model. Sequentially, the optimal learning rate and unit number determined by PSO intelligent optimization algorithm are input into the network for obtaining the optimal configuration of the model to predict thermally induced error. The flow chart of proposed PSO-LSTM model is shown in Fig. 3.

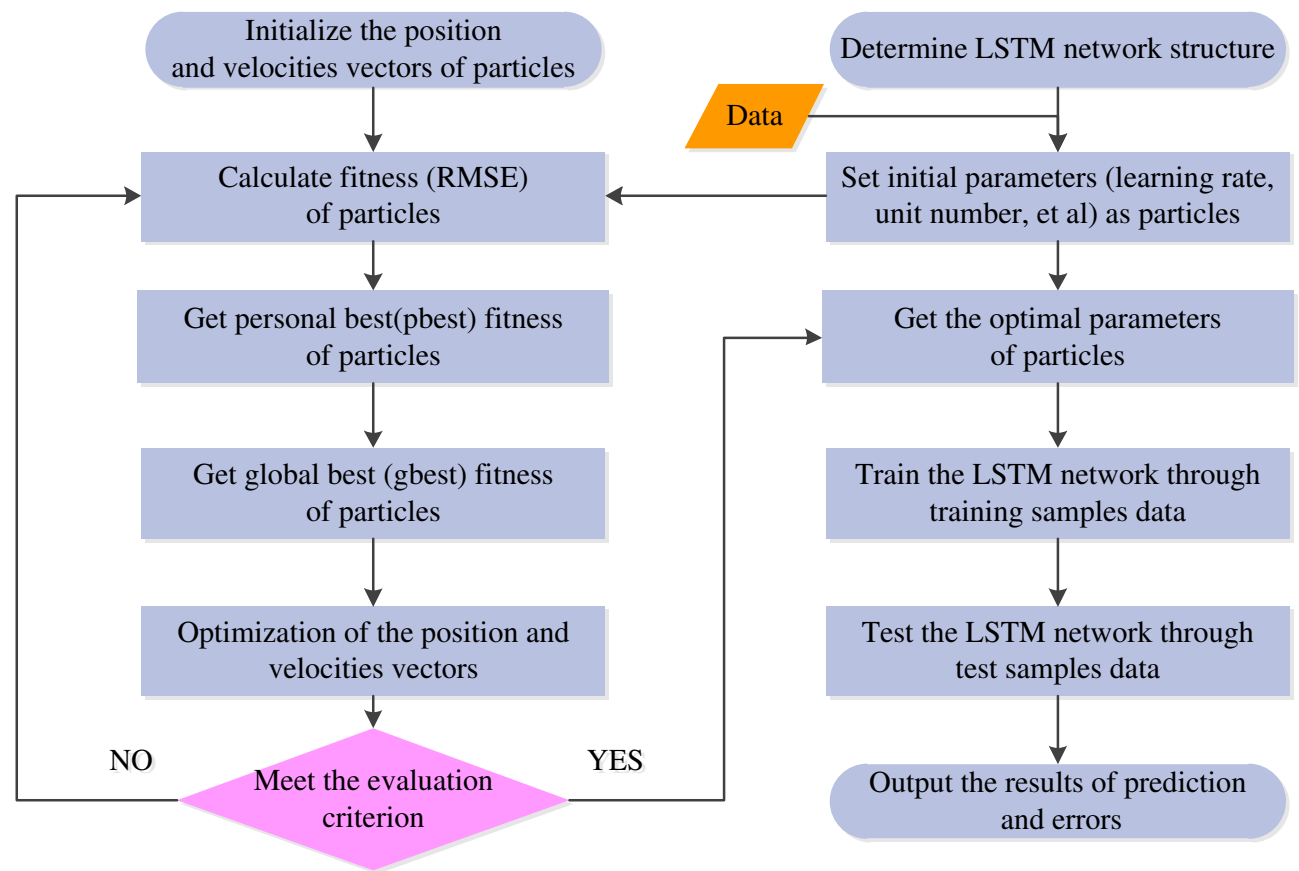

Fig. 3 Flow chart of the thermal error prediction modeling process

Software: Matlab R2019a, Deep learning and global optimization toolbox, Win7 64-bit operating system; Hardware: RAM 8 GB, Inter (R) Core (TM) i5-5200U CPU 2.20GHz.

\section{Results and Validation}

In order to validate the PSO-LSTM model for predicting thermally induced error of ball screws, thermal characteristic experiments for the time-series thermal error forecast are implemented in this paper. Firstly, thermal sensitive points of ball screws are captured according to thermal modal analysis (TMA) to determine the key temperature measurement point. Secondly, a thermal experiment with step speed of the motor for obtaining the data of temperature and deformation is carried out to validate the effectiveness of PSO-LSTM model, and then another thermal experiment with random speed is carried out to verify the robustness of the proposed model further. Finally, the performance of the proposed model is compared with that of RBF model and BP model.

\subsection{Thermal sensitive point}

Ball screws feed drive systems mainly include motor, coupling, bearings and ball screws. In the working status, the thermal deformation of ball screws is mainly caused by temperature rises of bearings, nuts and a screw. With the purpose of thermal error modeling, it is required to 
establish a correlation between the temperature rise of components and deformation. Therefore, the selection of temperature sensitive points based on thermal mode is introduced in this section. The thermal modes of the system represent the distribution of the temperature field under the corresponding eigenvalues, which is the reciprocal of the time constant. The corresponding transient response of temperature field can be acquired by superposition of each order thermal mode. Additionally, the areas with significant temperature changes can be identified rely on the shape of low order thermal modes. In order to analyze the thermal sensitive point of ball screws, a finite element (FE) model of ball screws is established and the thermal modal analysis is carried out.

To perform the thermal modal analysis, the finite element solution of the underlying heat transfer problem needs to be solved [12], which requires the integration of coupled differential equations of the form

$\left[C_{T}\right]\{T(t)\}+\left[K_{T}\right]\{T(t)\}=\{Q(t)\}$

Where $\left[C_{T}\right]$ and $\left[K_{T}\right]$ are the heat capacity matrix and the heat conductivity matrix, respectively, $\{T(t)\}$ is the nodal temperature vector, and $\{Q(t)\}$ is the nodal thermal load vector.

The eigen-problem associated with Eq. (13) is

$\left[K_{T}\right]\left[\Phi_{T}\right]=\left[C_{T}\right]\left[\Phi_{T}\right][\Lambda]$

Where $[\Lambda]$ is a diagonal matrix composed of all the eigenvalues, $\lambda_{i}$, and $\left[\Phi_{T}\right]$ are the corresponding eigenvector matrix. Theoretically, $i$ is the reciprocal of the corresponding time constant

$\lambda_{i}=\frac{1}{\tau_{i}}$

Where $\lambda_{i}$ and $\tau_{i}$ are the $i$-th eigenvalue and time constant, respectively. The time constant describes how quickly the mode responds to thermal loads.

The first four thermal modes with the time constants and the corresponding temperature field distributions are calculated by thermal modal analysis, the magnitudes of the temperature for each mode are normalized. The first four thermal modes with temperature fields and thermal time constant are shown in Fig. 4. It is seen that the thermal time constant of ball screws is greater, indicating that the variation of temperature field is slow. It is depicted significant temperature rise occurs on the fixed bearing, the support bearing, the nut/screw interface. Therefore, the temperature sensitive points are determined and three temperature measuring points are arranged on the surface of the nut and the end surface of two bearings in this research. 

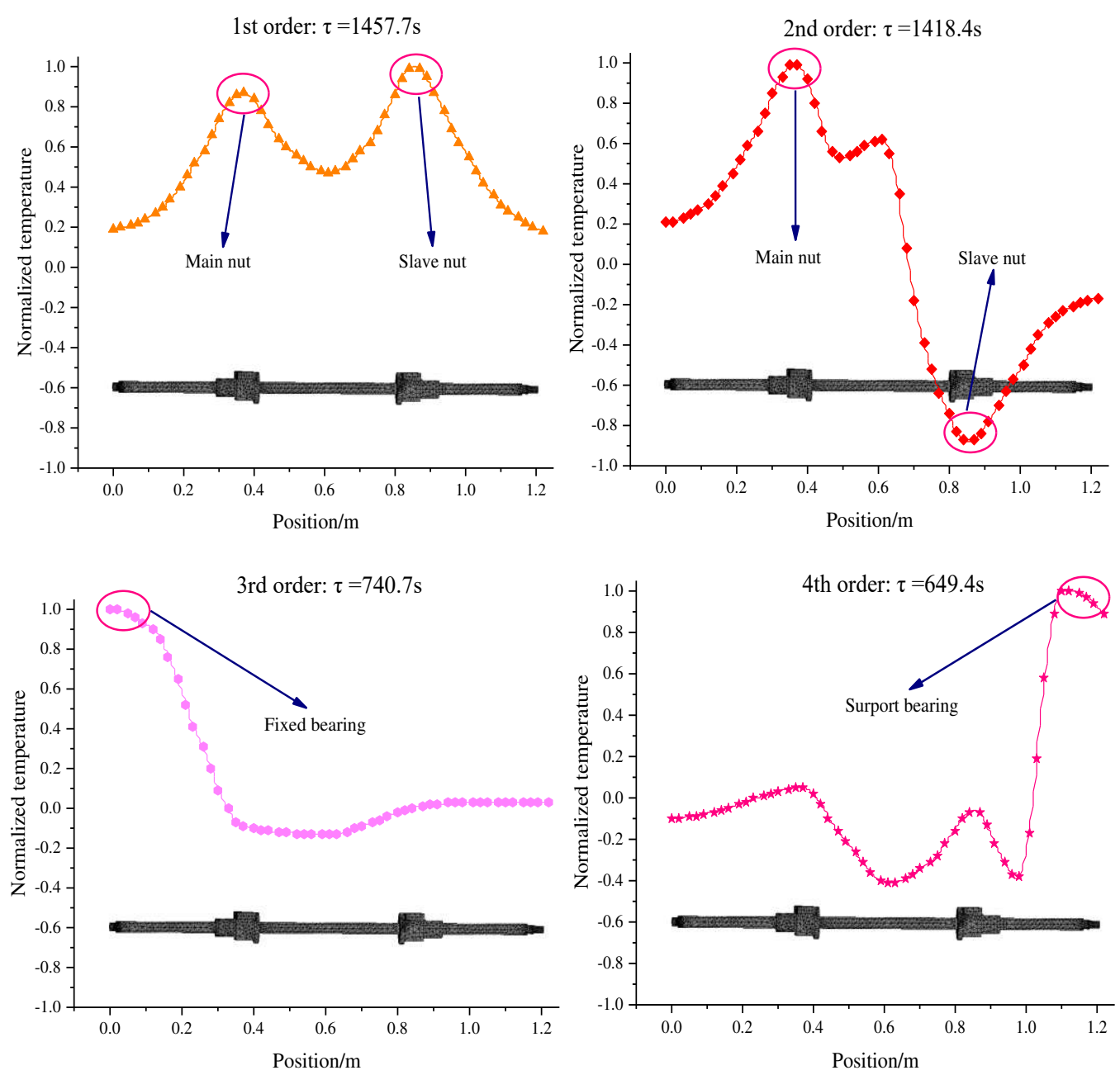

Fig. 4 First four thermal modes with temperature fields and time constants

\subsection{Experimental results and validation}

To verify the effectiveness and robustness of this proposed model and to compare with the performance of other models in this study, two thermal characteristic experiments with step speed and random speed are conducted on the high-speed precision ball screw test bench in our laboratory. The experimental setup is shown in Fig. 5(a-e). Parameters of the measuring instrument are shown in Table 1. Three temperature sensors are mounted on the feed drive system to obtain the temperature data, where they are arranged on the front bearing seating, the rear bearing seating and the nut, respectively. An eddy current displacement sensor is installed on the end face of the screw to measure the thermal deformation. Additionally, the ambient temperature is monitored during the experiment so as to consider the effect of ambient temperature on thermal deformation. 
Table 1 Parameters of experimental instrument.

\begin{tabular}{cc}
\hline Type & Product description \\
\hline Temperature sensors & KYW-TC, Kunlunyuanyang, Beijing, China \\
Eddy current displacement sensor & ML33-01-00-03, Milang, Shenzhen, China \\
Temperature inspection instrument & Kunlunyuanyang, Beijing, China \\
Displacement inspection instrument & XSAE-CHVB1M2V0, Milang, Shenzhen, China \\
\hline
\end{tabular}

First of all, the ball screw runs as reciprocating cycle at a rotational speed of 500r/min for 10min, where the purpose is to avoid the influence of gap between the parts of ball screws and instability of the machine at the startup stage, and the accuracy of experimental results is guaranteed. Ten minutes later the experiment is carried out according to the speed spectrum and the nut moves along with the screw as the reciprocating cycle. The temperature of the front bearing housing, the rear bearing housing and the nut, the ambient temperature and the thermal deformation of the screw are in real-time collected where the data is recorded by a self-designed data acquisition system and sampling period assigned as $600 \mathrm{~ms}$. After the experiment, the temperature of components should be naturally cooled to the ambient temperature and then another experiment continues.

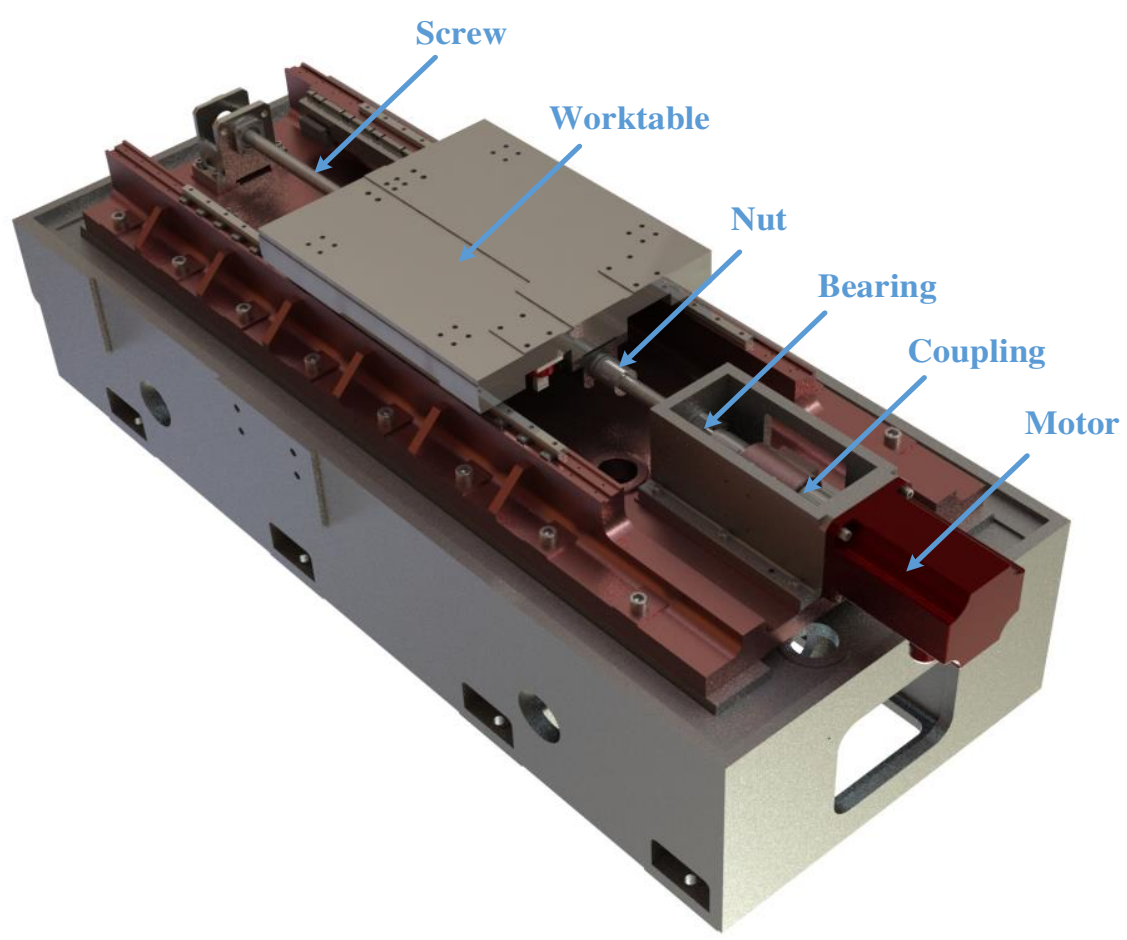

(a) Test bench of ball screws 


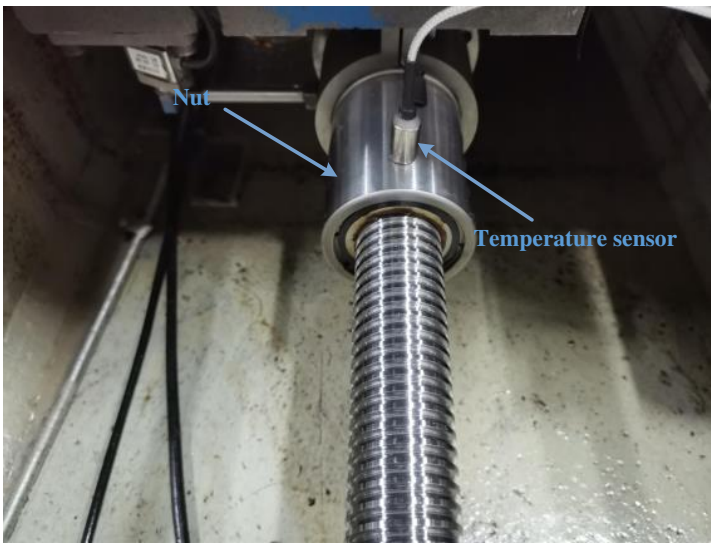

(b) Temperature sensor mounted on nut

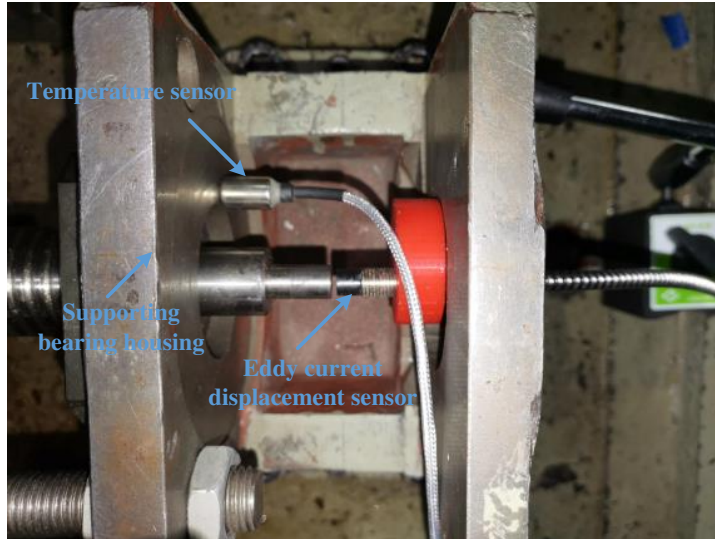

(d) Temperature and thermal deformation test

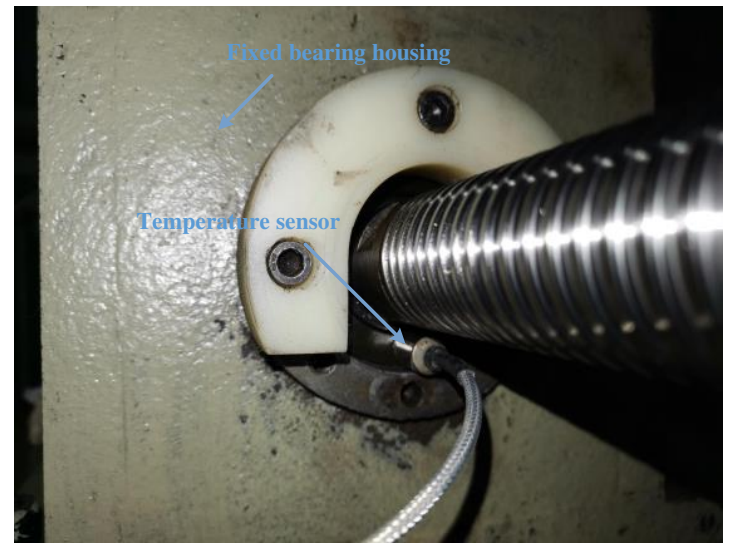

(c) Temperature sensor mounted near fixed bearing

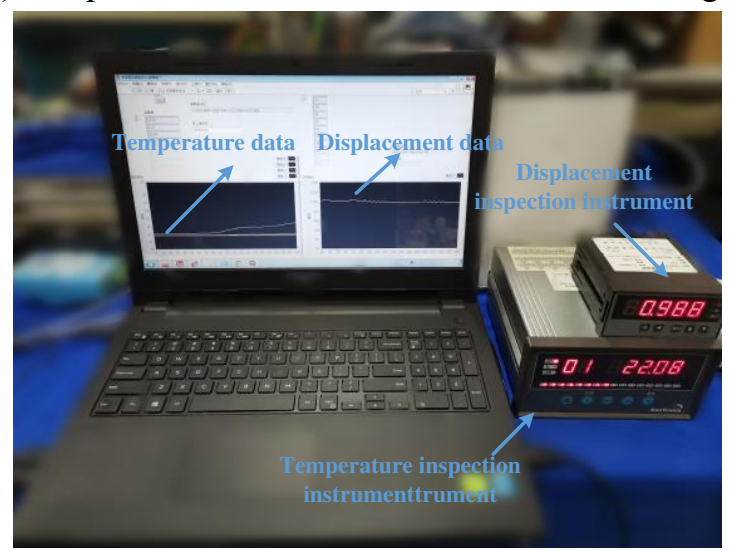

(e) Data acquisition system

Fig. 5 Experiment setup

A thermal characteristic experiment with step speed of the motor is carried out to validate the effectiveness of PSO-LSTM model. The speed of the motor is changed on every 20 minutes and increases from 300r/min to $1000 \mathrm{r} / \mathrm{min}$ then decreases to $300 \mathrm{r} / \mathrm{min}$ (See Fig. 6). The experimental result based on step speed is shown in Fig. 7. According to experimental results, the data of temperature rise and thermal deformation is normalized and feed the neural network models. The first $80 \%$ data is taken as a training set and the last $20 \%$ data is assigned as a testing set. The PSO searching result shows the time window size, learning rate and unit number is 5 , 0.0037 and 88 respectively. In order to verify the advantage of this proposed model, the results of this model is compared with that of other models. The parameters of each network model are fairly assigned. Comparative results of each model under the step speed are shown in Fig. 8. It is clear that the result of PSO-LSTM model is almost entirely consistent with the experimental result. The RBF and BP can reasonably predict the values that never appeared, but the error between predictive result and experimental result of both two models is greater than PSO-LSTM one. The result of the testing set can indicate the performance of model and is most critical for validating the model. Fig. 9 illustrates the relative error distribution characteristics of the three models. It is concluded that the results of PSO-LSTM model and BP model are better than RBF model. The PSO-LSTM model and BP model are both with high accuracy while the PSO-LSTM is more accurate than the BP model. Fig. 10 presents the absolute error distribution characteristics of the three methods. It can be found that many error points of RBF and BP are beyond $10 \mu \mathrm{m}$ and some of them even close to 20-30 $\mu \mathrm{m}$. In contrast, the absolute error of data obtained from PSO-LSTM are mostly controlled within $5 \mu \mathrm{m}$, only a little of points outside of $5 \mu \mathrm{m}$ but not larger than $8 \mu \mathrm{m}$. 
Fig. 11 shows the results of regression state analysis for the three models. The regression prediction performance of PSO-LSTM model is better than others. To quantify the predictive performance of the models, the four criterias, RMSE, MAE, MSE and MAPE of the testing set of the three models are listed in Table 2. It is concluded that the PSO-LSTM model has the smallest RMSE, MAE, MSE and MAPE with the highest precision, followed by the BP model, and then the RBF model. Consequently, the proposed model provides an exact global trend prediction of thermal error, meanwhile the thermal error at a certain time also can be accurately determined. As the predictive results well agree with the experimental one, the PSO-LSTM model is validated. Via comparisons with other models, It is concluded that the PSO-LSTM model has the advantage in prediction accuracy.

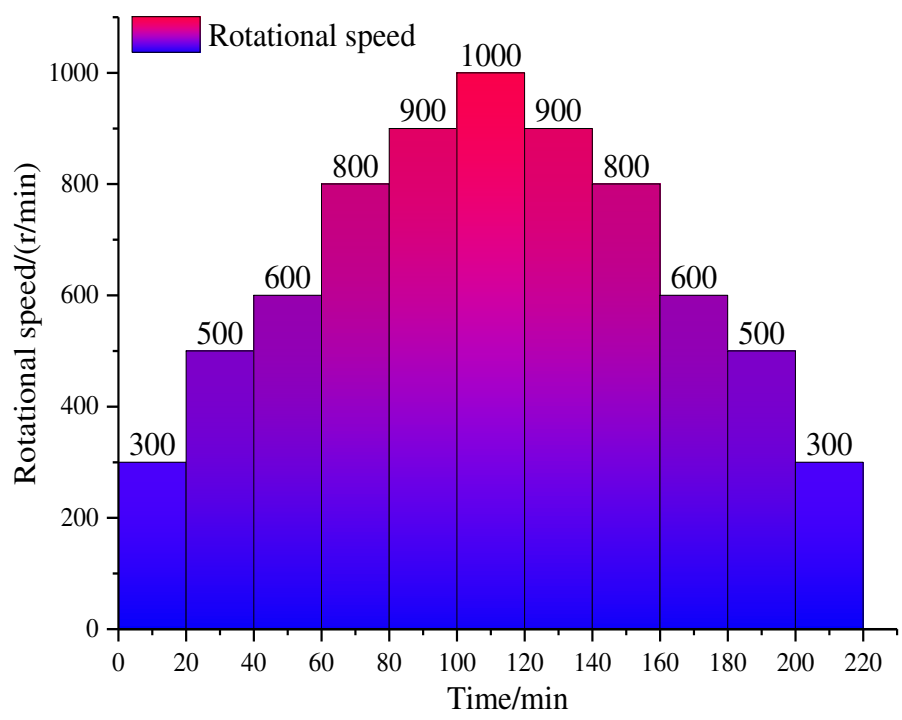

Fig. 6 Step speed spectrum

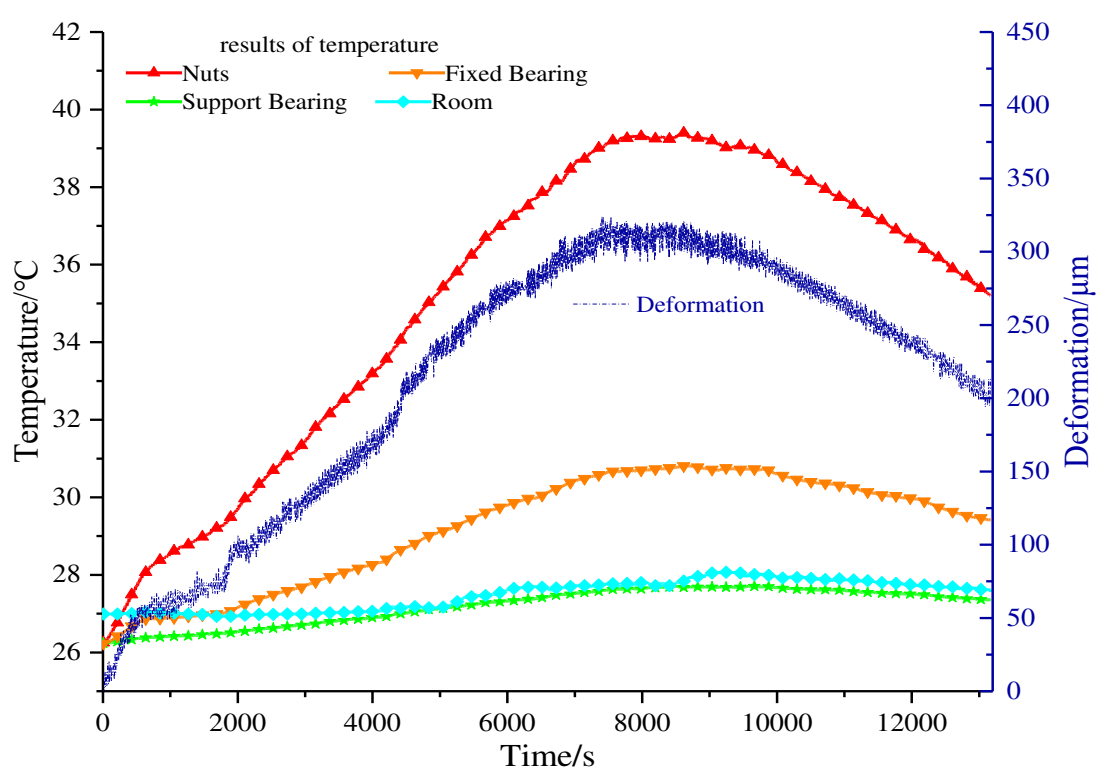

Fig. 7 Experimental results of thermal error in step speed 


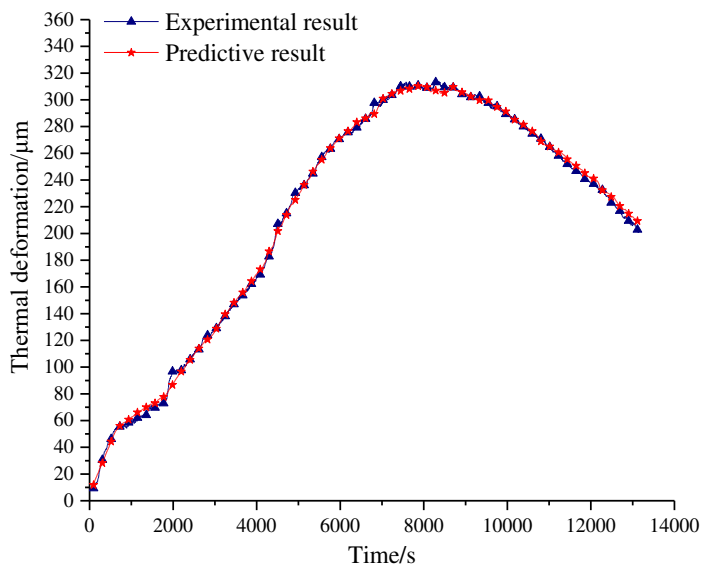

(a) Predictive results of the PSO-LSTM model

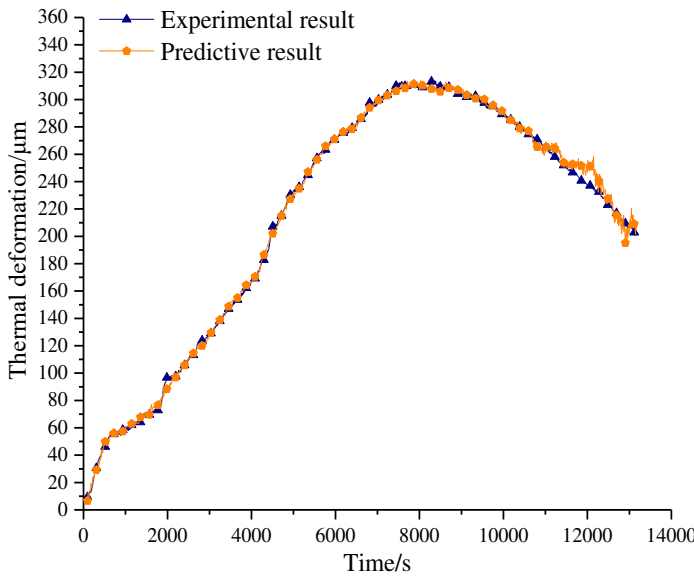

(b) Predictive results of the RBF model

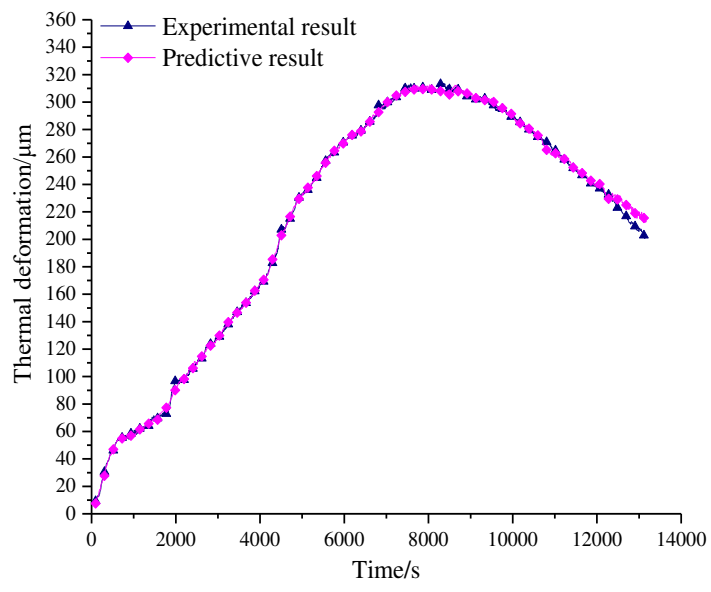

(c) The predictive results of the BP model

Fig. 8 Comparative results between the experiment and the prediction of the three models

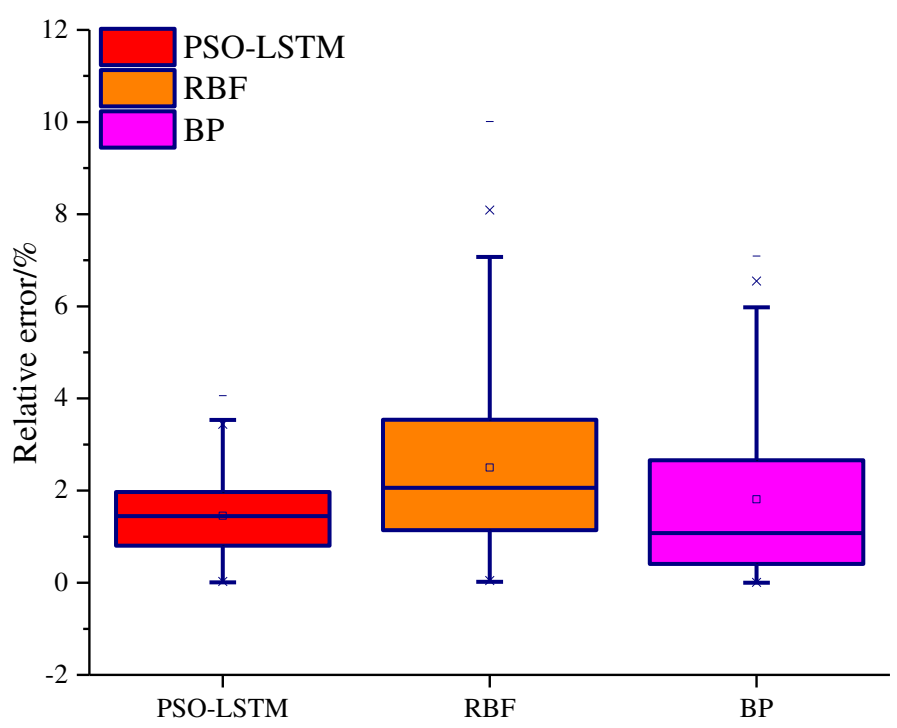

Fig. 9 Absolute relative error of the three models 


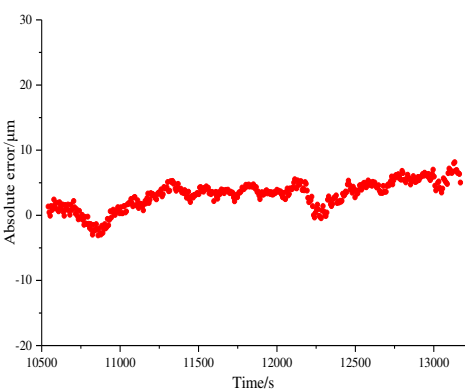

(a) PSO-LSTM model

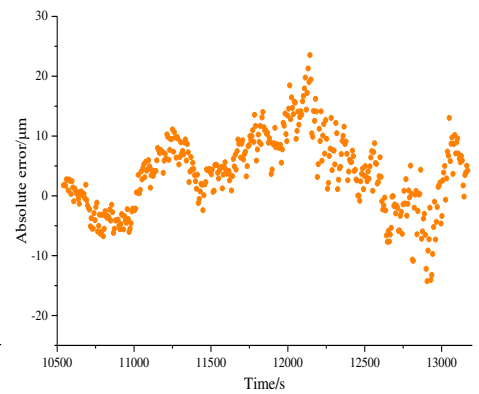

(b) RBF model

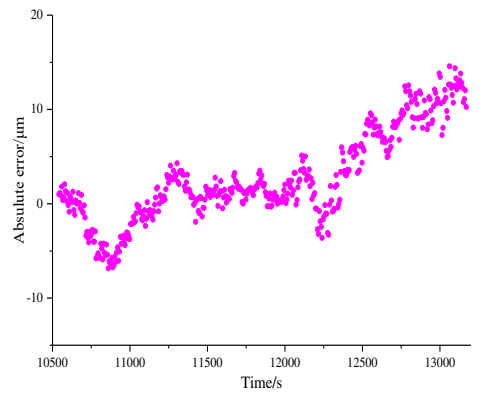

(c) BP model

Fig. 10 Absolute error scatterplot of the testing set

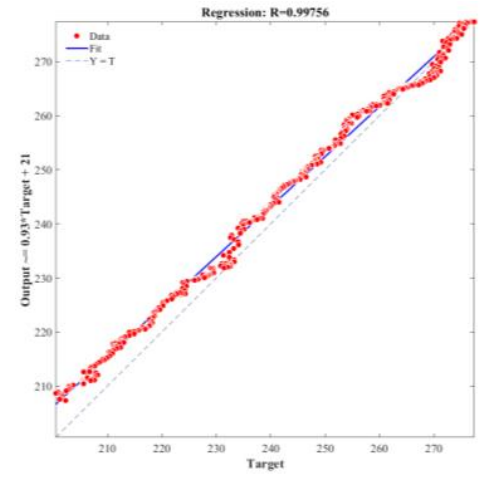

(a) PSO-LSTM model

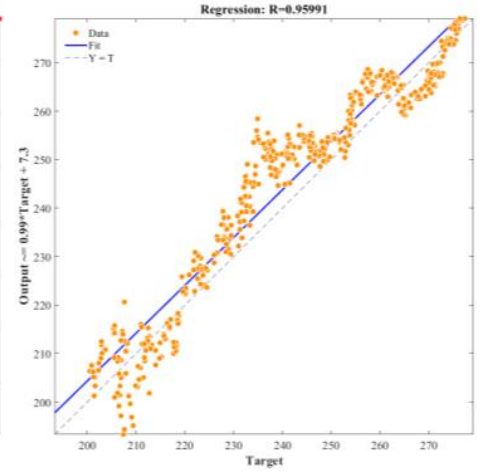

(b) RBF model

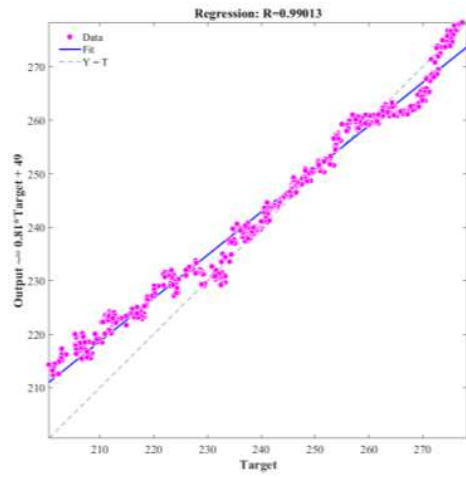

(c) BP model

Fig. 11 Regression analysis

Table 2 Comparison among different predictive models

\begin{tabular}{ccccc}
\hline Models & RMSE & MAE & MSE & MAPE \\
\hline PSO-LSTM & 3.7755 & 3.5557 & 14.2541 & 1.4281 \\
RBF & 7.2715 & 5.9230 & 52.8746 & 2.4339 \\
BP & 5.5459 & 4.0659 & 30.7569 & 1.7565 \\
\hline
\end{tabular}

According to the random speed spectrum (See Fig. 12), another thermal experiment is carried out to verify the robustness of the proposed model. The experimental results based on step speed are shown in Fig. 13. The experimental data is normalized and imported to the neural network. The first $80 \%$ data is taken as a training set and the last $20 \%$ data is assigned as a testing set. The PSO searches that the time window size is 5, the learning rate is 0.0029 and the unit number is 86 . In order to compare fairly, $\mathrm{RBF}$ and $\mathrm{BP}$ are set with the same structure. The prediction results of three models are shown in Fig. 14. It can be seen from Fig. 14 that the LSTM model outperform others, and the RBF model and the BP model have an obvious decline in their accuracy. Although the results of $\mathrm{RBF}$ and $\mathrm{BP}$ are acceptable for the training set, a certain data of the testing set are with large error and even some of them close to $6 \%$. The results of RBF and BP for the testing set are both with obvious errors, which indicate that the generalizability is not strong enough to predict the data that never appear in the training stage. In contrast, the proposed PSO-LSTM model can predict the thermal error variation more accurately.

The relative error obtained by the PSO-LSTM model is smaller than that of other two models, as depicted in Fig. 15. The absolute error distribution characteristics of the three methods are shown in Fig. 16. It is concluded that the PSO-LSTM model has a smaller and narrower error 
band. Most error points of RBF and BP in testing set are beyond $10 \mu \mathrm{m}$ and some of them even close to $20 \mu \mathrm{m}$. In contrast, the absolute error of data from PSO-LSTM is all controlled within $5 \mu \mathrm{m}$, which shows the excellent performance of this proposed model. The results of regression state analysis for the three models are shown in Fig. 17. The regression prediction performance of PSO-LSTM model is better than others. The statistic evaluation indexes of the testing set of the three models are given in Table 3. The PSO-LSTM model still has the smallest RMSE, MAE, MSE and MAPE with the highest precision. The PSO-LSTM model has higher accuracy and lower error compared with the RBF model and BP model in two thermal characteristic experiments based on step speed and random speed. The generalization ability of the PSO-LSTM model is superior to the other two models according to the experimental results. The proposed model can not only provide an accuracy predictive result of thermal error but also maintain a stable and satisfactory performance even in complex work condition. Therefore, the effectiveness and robustness of this proposed model are verified. The proposed method in this paper can be implemented to predict the thermal error of ball screws, and provide a foundation for thermal error compensation.

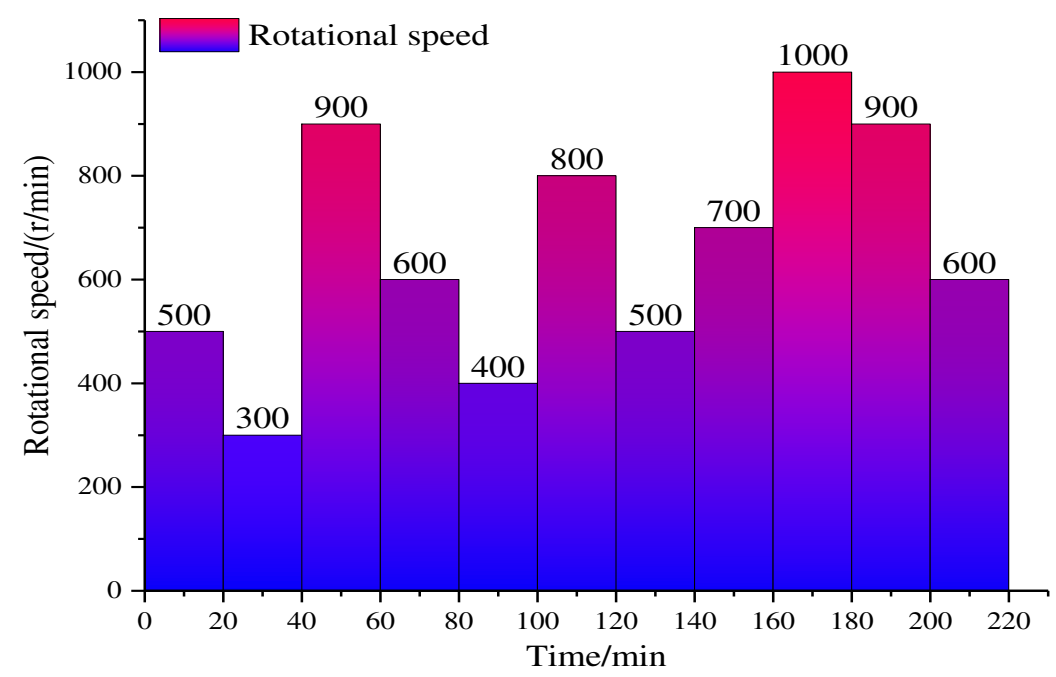

Fig. 12 The random speed spectrum

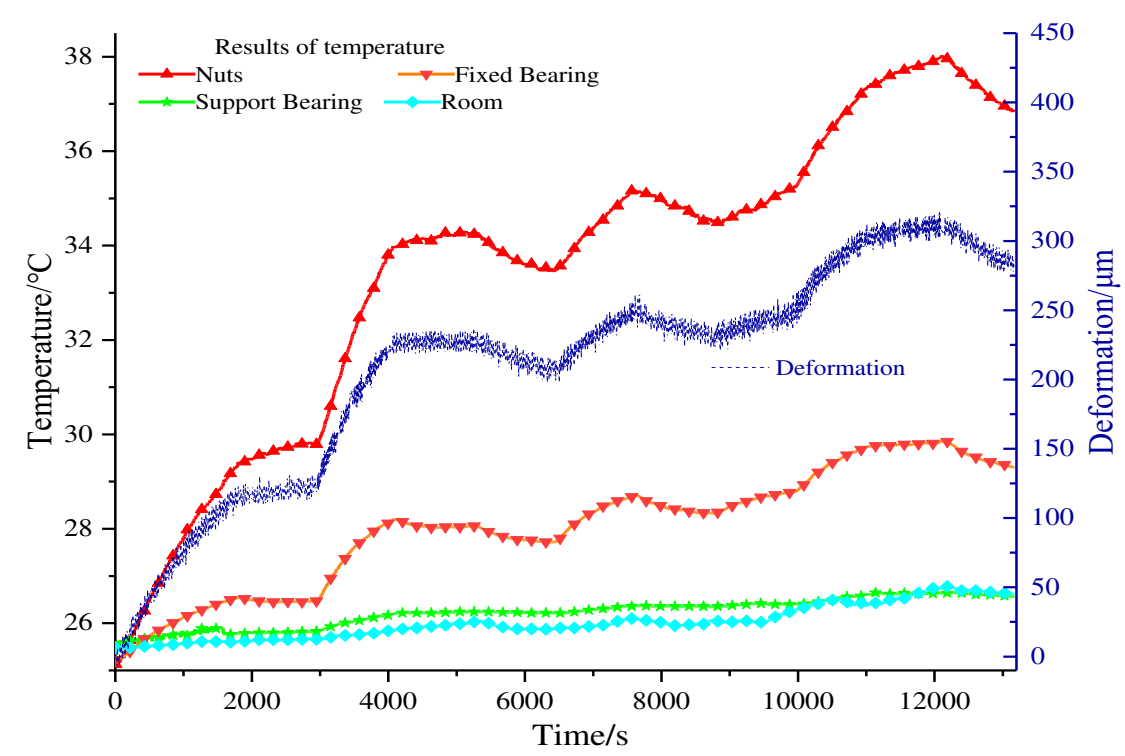

Fig. 13 Experimental results of thermal error in random speed 

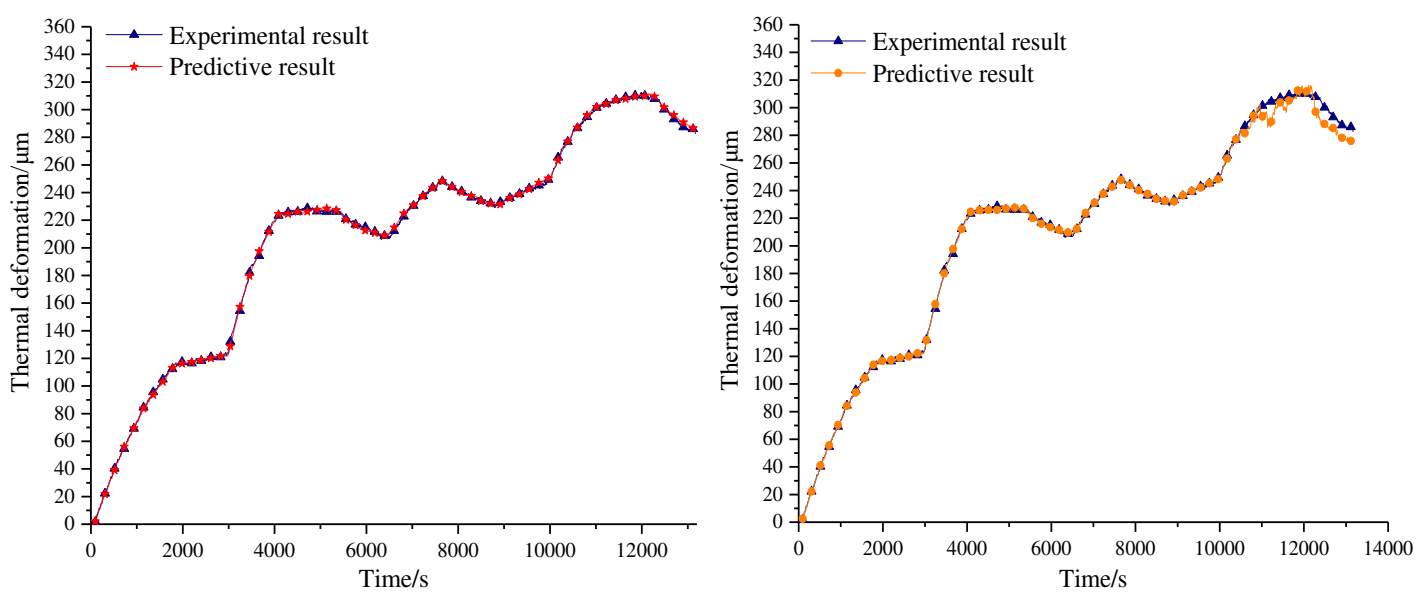

(a) The predictive results of the PSO-LSTM model (b) The predictive results of the RBF model

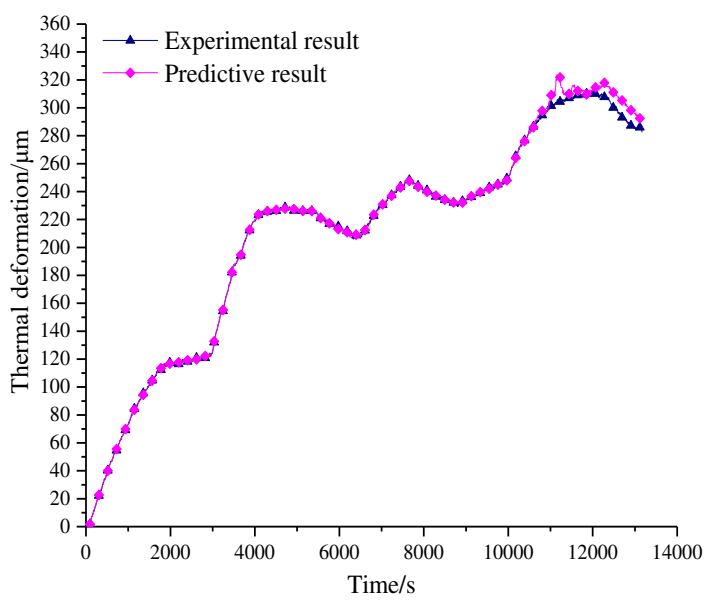

(c) The predictive results of the BP model

Fig. 14 Comparative results between the experiment and the prediction of the three models.

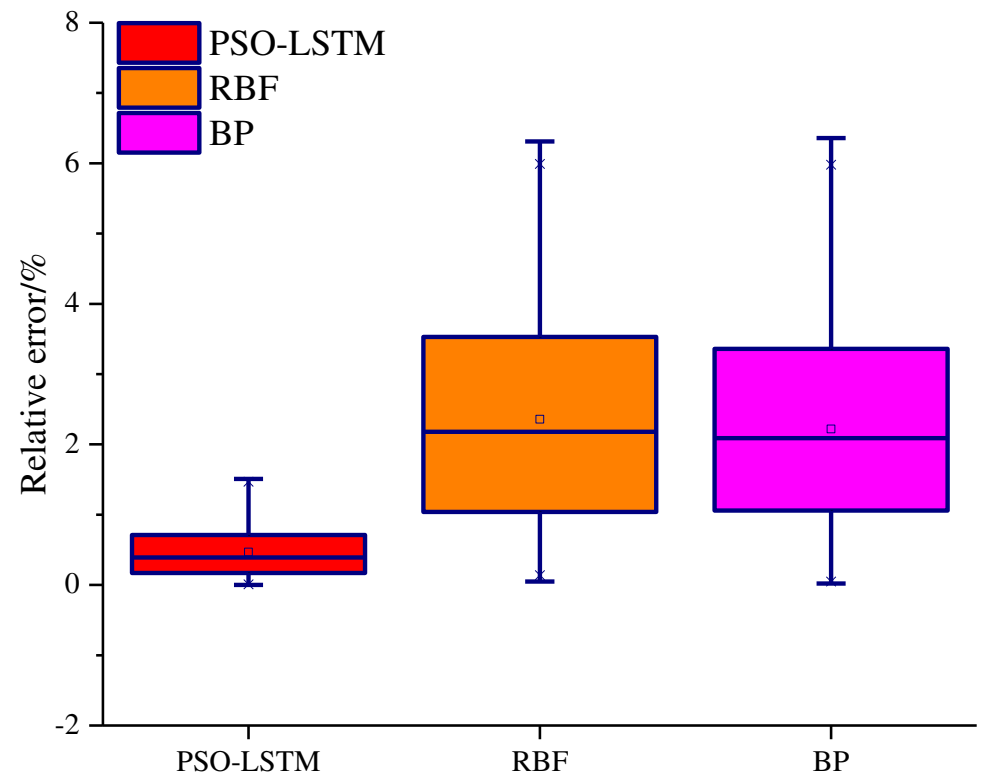

Fig.15 Absolute relative error of the three models 


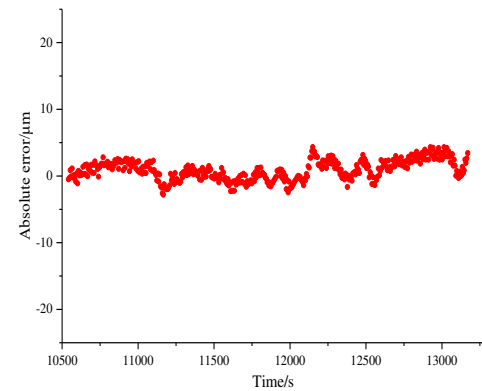

(a) PSO-LSTM model

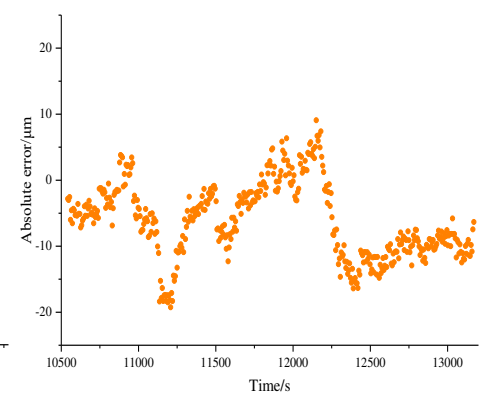

(b) RBF model

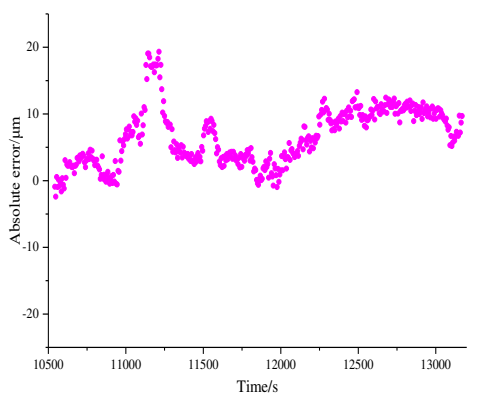

(c) BP model

Fig. 16 Absolute error scatterplot the testing set

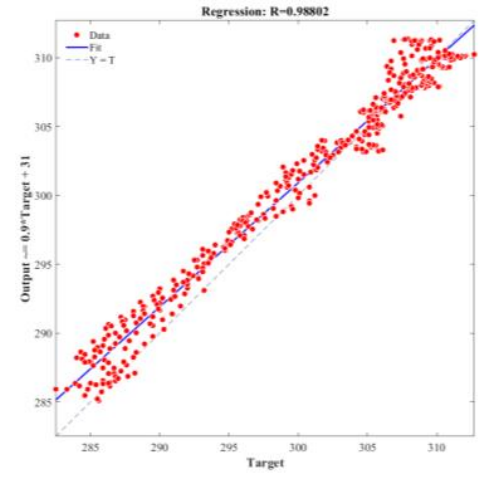

(a) PSO-LSTM model

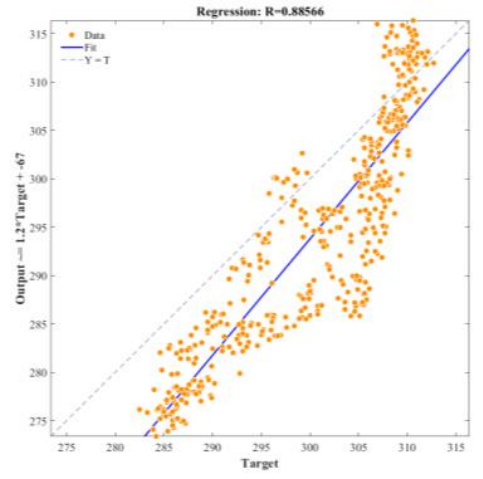

(b) RBF model

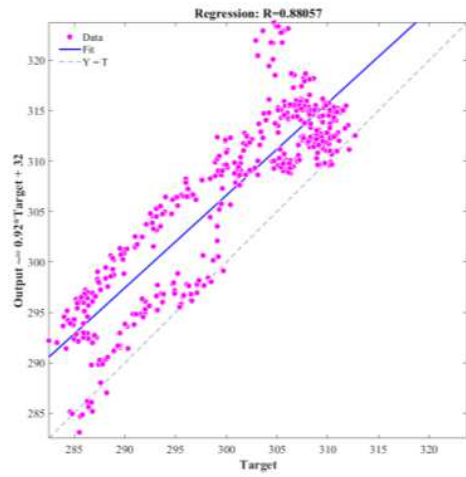

(c) BP model

Fig. 17 Regression analysis curve

Table 3 Comparison among different prediction models

\begin{tabular}{ccccc}
\hline Models & RMSE & MAE & MSE & MAPE \\
\hline PSO-LSTM & 1.7217 & 1.3866 & 2.9644 & 0.4636 \\
RBF & 8.3665 & 7.0474 & 69.9985 & 2.4349 \\
BP & 7.8532 & 6.6345 & 61.6720 & 2.1513 \\
\hline
\end{tabular}

\section{Conclusions and future work}

In this paper, a novel data-driven model based on PSO-LSTM is proposed for predicting thermally induced error of ball screws, where the deep learning model combining with intelligent optimization algorithm is established based on experimental results. The thermal error of ball screws can be accurately predicted based on this proposed method, which provides a foundation for thermal error compensation to improve the machining accuracy. The effectiveness and robustness of this proposed model are validated by thermal error obtained from experiments based on step speed and random speed. The comparison between the proposed model and traditional models is implemented. The conclusions can be drawn as follow.

(1) A novel data-driven thermal errors model of ball screws based on PSO-LSTM is proposed, and the effectiveness of this model is validated by a thermal characteristic experiment at step speed on a self-designed ball screw test bench. Comparison between PSO-LSTM model and experiment shows that thermal error of ball screws can be accurately predicted by this proposed model, which can provide a foundation for thermal error compensation.

(2) Robustness of this proposed model is verified based on another thermal characteristic 
experiment at random speed. By comparison between predictive results and experimental ones, the PSO-LSTM model can not only provide an accuracy predictive result of thermal error but also maintain a stable and satisfactory robustness even in complex work conditions.

(3) Comparative results between this proposed model and the traditional models are analyzed. In terms of predicting thermally induced error of ball screws in this paper, it reveals that the PSO-LSTM model has higher accuracy and lower error compared with the RBF model and BP model, and has the smallest RMSE, MAE, MSE and MAPE. It is concluded that the PSO-LSTM model outperforms the others.

Although the predictive thermal error model of ball screws is established, the effect of thermal error compensation hasn't been checked yet. The thermal compensation based on this proposed model will be conducted and its effects will be checked in the next stage of our research work.

\section{Declarations}

Authors' Contributions Xiangsheng Gao conceived the experiment and modeling, and wrote the manuscript as well. Yueyang Guo conducted the experiment and modeling. Dzonu Ambrose Hanson conducted the data analysis and the English editing. Zhihao Liu conducted the experiment. Min Wang and Tao Zan supervised this work and revised the manuscript.

Funding The author(s) disclosed receipt of the following financial support for the research, authorship, and/or publication of this article: This study was supported by the National Natural Science Foundation of China (grant numbers: 51875008, 51505012 and 51575014).

Ethical Approval The article follows the guidelines of the Committee on Publication Ethics (COPE) and involves no studies on human or animal subjects.

Consent to Participate Not applicable.

Consent to Publish Not applicable.

Competing Interests The authors declare no competing interests.

Availability of Data and Materials All data generated or analyzed in this study are included in the present article.

\section{References}

1. W.S. Yun, S.K. Kim, D.W. Cho (1999) Thermal error analysis for a CNC lathe feed drive system. Int J Mach Tools Manuf 39: 1087-1101

2. J. Bryan (1990) International status of thermal error research. CIRP Annals-Manuf Technol 39: 645-656

3. P.C. Tsai, C.C. Cheng, Y.C. Hwang (2014) Ball screw preload loss detection using ball pass frequency. Mech Syst Signal Process 48: 77-91 
4. Z.Z. Xu, X.J. Liu, H.K. Kim, J.H. Shin, S.K. Lyu (2011) Thermal error forecast and performance evaluation for an air-cooling ball screw system. Int J Mach Tools Manuf 51: $605-611$

5. Z.Z. Xu, X.J. Liu, C.H. Choi, S.K. Lyu (2012) A study on improvement of ball screw system positioning error with liquid-cooling. Int J Precis Eng Manuf 13: 2173-2181

6. H. Shi, B. He, Y. Yue, C. Min, X. Mei (2019) Cooling effect and temperature regulation of oil cooling system for ball screw feed drive system of precision machine tool. Appl Therm Eng 161: 114150

7. X.S. Gao, Z.Y. Qin, Y.Y. Guo, M. Wang, T. Zan (2019) Adaptive method to reduce thermal deformation of ball screws based on carbon fiber reinforced plastics. Materials 12: 3113

8. Y.Y. Guo, X.S. Gao, M. Wang, T. Zan (2020) Bio-inspired graphene-coated ball screws: Novel approach to reduce the thermal deformation of ball screws. Proc Inst Mech Eng Part C J Mech Eng Sci 0: 1-11

9. S. Yang, J. Yuan, J. Ni (1996) The improvement of thermal error modeling and compensation on machine tools by CMAC neural network. Int J Mach Tools Manuf 36: 527-537

10. J.G. Yang, J.X. Yuan, J. Ni (1999) Thermal error mode analysis and robust modeling for error compensation on a CNC turning center. Int J Mach Tools Manuf 39: 1367-1381

11. H.T. Zhao, J.G. Yang, J.H. Shen (2007) Simulation of thermal behavior of a CNC machine tool spindle. Int J Mach Tools Manuf 47: 1003-1010

12. J. Zhu, J. Ni, A.J. Shih (2008) Robust Machine Tool Thermal Error Modeling Through Thermal Mode Concept. J Manuf Sci E-T ASME 130 (6): 061006

13. T.J. Li, C.Y. Zhao, Y.M. Zhang (2018) Adaptive real-time model on thermal error of ball screw feed drive systems of CNC machine tools. Int J Adv Manuf Technol 94: 3853-3861

14. H. Shi, C. Ma, J. Yang (2015) Investigation into effect of thermal expansion on thermally induced error of ball screw feed drive system of precision machine tools. Int J Mach Tool Manuf 97: 60-71

15. R. Ramesh, M.A. Mannan, A.N. Poo (2003) Thermal error measurement and modelling in machine tools. : Part I. Influence of varying operation condition. Int J Mach Tools Manuf 43: 391-404

16. R. Ramesh, M.A. Mannan, A.N. Poo, S.S. Keerthi (2003) Thermal error measurement and modelling in machine tools, : Part II. Hybrid Bayesian Network - Support vector machine model. Int J Mach Tools Manuf 43: 405-419

17. H. Wu, H.T. Zhang, Q.J. Guo, X.S. Wang, J.G. Yang (2008) Thermal error optimization modeling and real-time compensation on a CNC turning center. J Mater Process Technol 207: $172-179$

18. Y. Zhang, J.G. Yang, H. Jiang (2012) Machine tool thermal error modeling and prediction by grey neural network. Int J Adv Manuf Technol 59: 1065-1072

19. H.T. Wang, L.P. Wang, T.M. Li, J. Han (2013) Thermal sensor selection for the thermal error modeling of machine tool based on the fuzzy clustering method. Int J Adv Manuf Technol 69: $121-126$

20. E.M. Miao, Y.Y. Gong, L.C. Dang, J.C. Miao (2014) Temperature-sensitive point selection of thermal error model of CNC machining center. Int J Adv Manuf Technol 74: 681-691

21. E.M. Miao, Y.Y. Gong, P.C. Niu, C.Z. Ji, H.D. Chen (2013) Robustness of thermal error compensation modeling models of CNC machine tools. Int J Adv Manuf Technol 69: 
2593-2603

22. J. Yang, H. Shi, B. Feng, L. Zhao, C. Ma, X. Mei (2014) Applying neural network based on fuzzy cluster pre-processing to thermal error modeling for coordinate boring machine. Procedia CIRP 17: 698-703

23. A. M. Abdulshahed, A. Longstaff, S. Fletcher, A. Myers (2013) Comparative study of ANN and ANFIS prediction models for thermal error compensation on CNC machine tools, in: Laser Metrology and Machine Performance X, Buckinghamshire. 79-89

24. A.M. Abdulshahed, A.P. Longstaff, S. Fletcher (2015) The application of ANFIS prediction models for thermal error compensation on CNC machine tools. Appl Soft Comput 27: $158-168$

25. H. Liu, E.M. Miao, X.Y. Wei, X.D. Zhuang (2017) Robust modeling method for thermal error of CNC machine tools based on ridge regression algorithm. Int J Mach Tools Manuf 113: $35-48$

26. M.O. Dos Santos, G.F. Batalha, E.C. Bordinassi, G.F. Miori (2018) Numerical and experimental modeling of thermal errors in a five-axis CNC machining center. Int $\mathrm{J}$ Adv Manuf Technol 96: 2619-2642

27. Y.Q. Huang, J. Zhang, X. Li, L.J. Tian (2014) Thermal error modeling by integrating GA and BP algorithms for the high-speed spindle. Int J Adv Manuf Technol 71: 1669-1675

28. I. Rojek, M. Kowal, A. Stoic, Predictive compensation of thermal deformations of ball screws in cnc machines using neural networks, Teh. Vjesn. 24 (2017) 1697-1703.

29. B. Li, Y. Zhang, L. Wang, X. Li (2019) Modeling for CNC Machine tool thermal error based on genetic algorithm optimization wavelet neural networks. Journal Mech Eng 55: 215-220 (In Chinese)

30. A. Sagheer, M. Kotb (2019) Time series forecasting of petroleum production using deep LSTM recurrent networks. Neurocomputing 323: 203-213

31. Y.Z. Zhang, R. Xiong, H.W. He, M.G. Pecht (2018) Long short-term memory recurrent neural network for remaining useful life prediction of lithium-ion batteries. IEEE Trans Veh Technol 67: 5695-5705

32. J.F. Zhang, Y. Zhu, X.P. Zhang, M. Ye, J.Z. Yang (2018) Developing a long short-term memory (LSTM) based model for predicting water table depth in agricultural areas. J Hydrol 561: 918-929

33. Y. Qin, S. Xiang, Y. Chai, H.Z. Chen (2020) Macroscopic-microscopic attention in LSTM networks Based on fusion features for gear remaining life prediction. IEEE Trans Ind Electron 67: 10865-10875

34. Y. Lecun, Y. Bengio, G. Hinton (2015) Deep learning. Nature 521: 436-444

35. R. Zhao, R.Q. Yan, Z.H. Chen, K.Z. Mao, P. Wang, R.X. Gao (2019) Deep learning and its applications to machine health monitoring. Mech Syst Signal Proc 115: 213-237

36. R. Yang, S.K. Singh, M. Tavakkoli, N. Amiri, Y. Yang, M.A. Karami, R. Rai (2020) CNN-LSTM deep learning architecture for computer vision-based modal frequency detection. Mech Syst Signal Process 144: 106885

37. R.B. Sun, Z.B. Yang, L.D. Yang, B.J. Qiao, X.F. Chen, K. Gryllias (2020) Planetary gearbox spectral modeling based on the hybrid method of dynamics and LSTM. Mech Syst Signal Process 138: 106611

38. K. Greff, R.K. Srivastava, J. Koutnik, B.R. Steunebrink, J. Schmidhuber (2017) LSTM: A 
search space odyssey. IEEE Trans Neural Networks Learn Syst 28: 2222-2232 


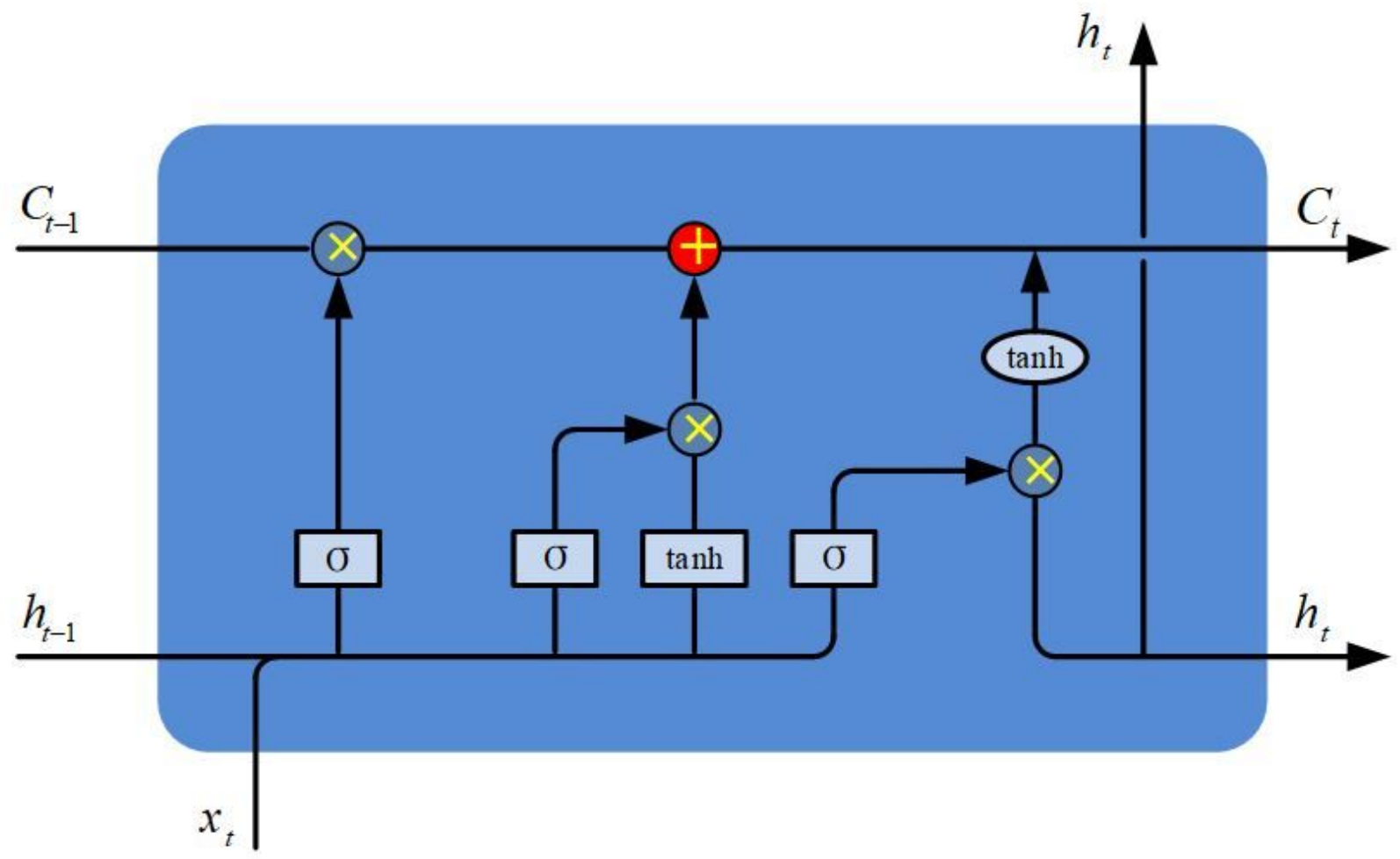

Figure 1

Framework of the LSTM neural network

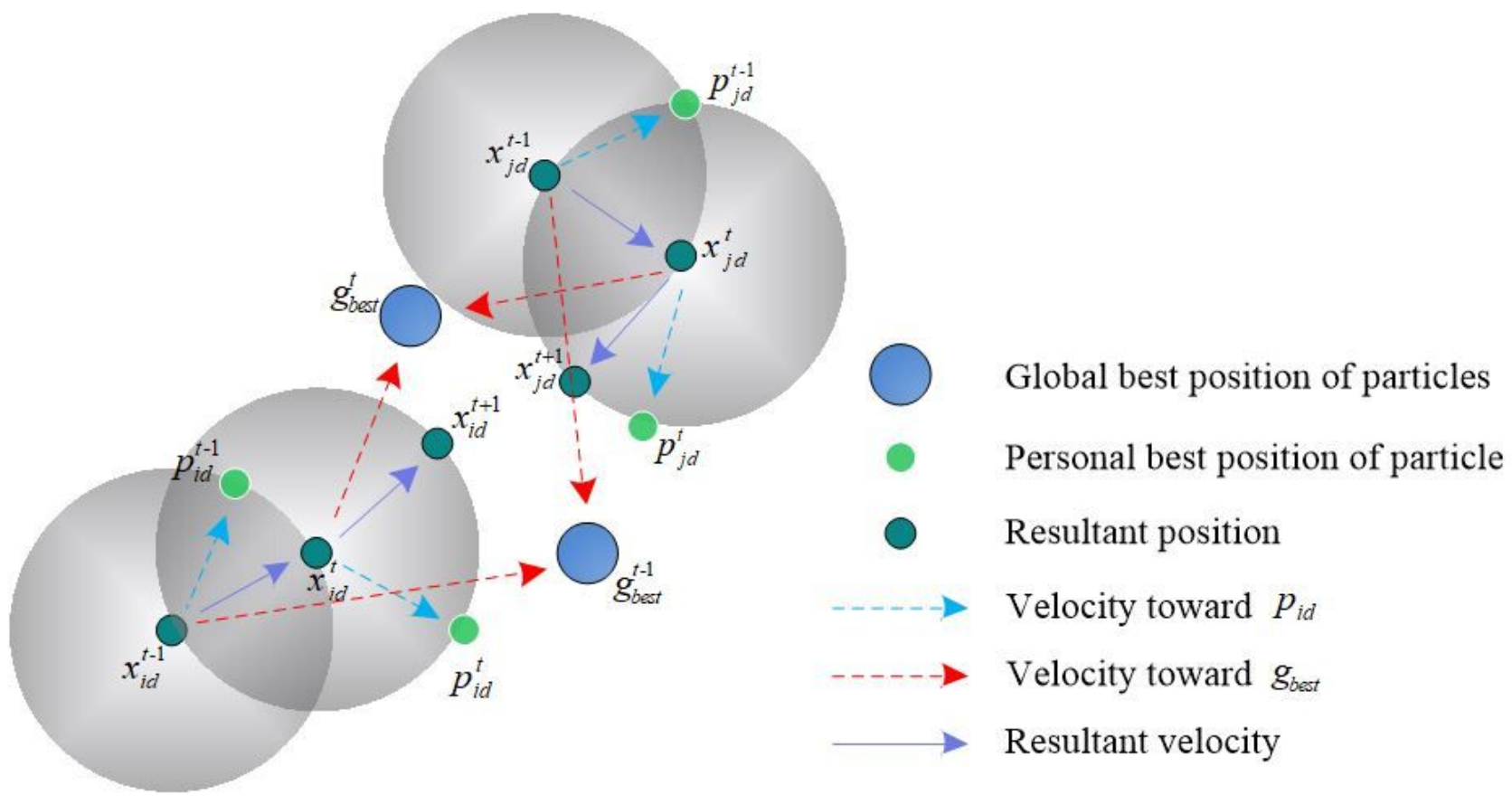

Figure 2

Search process of particles 


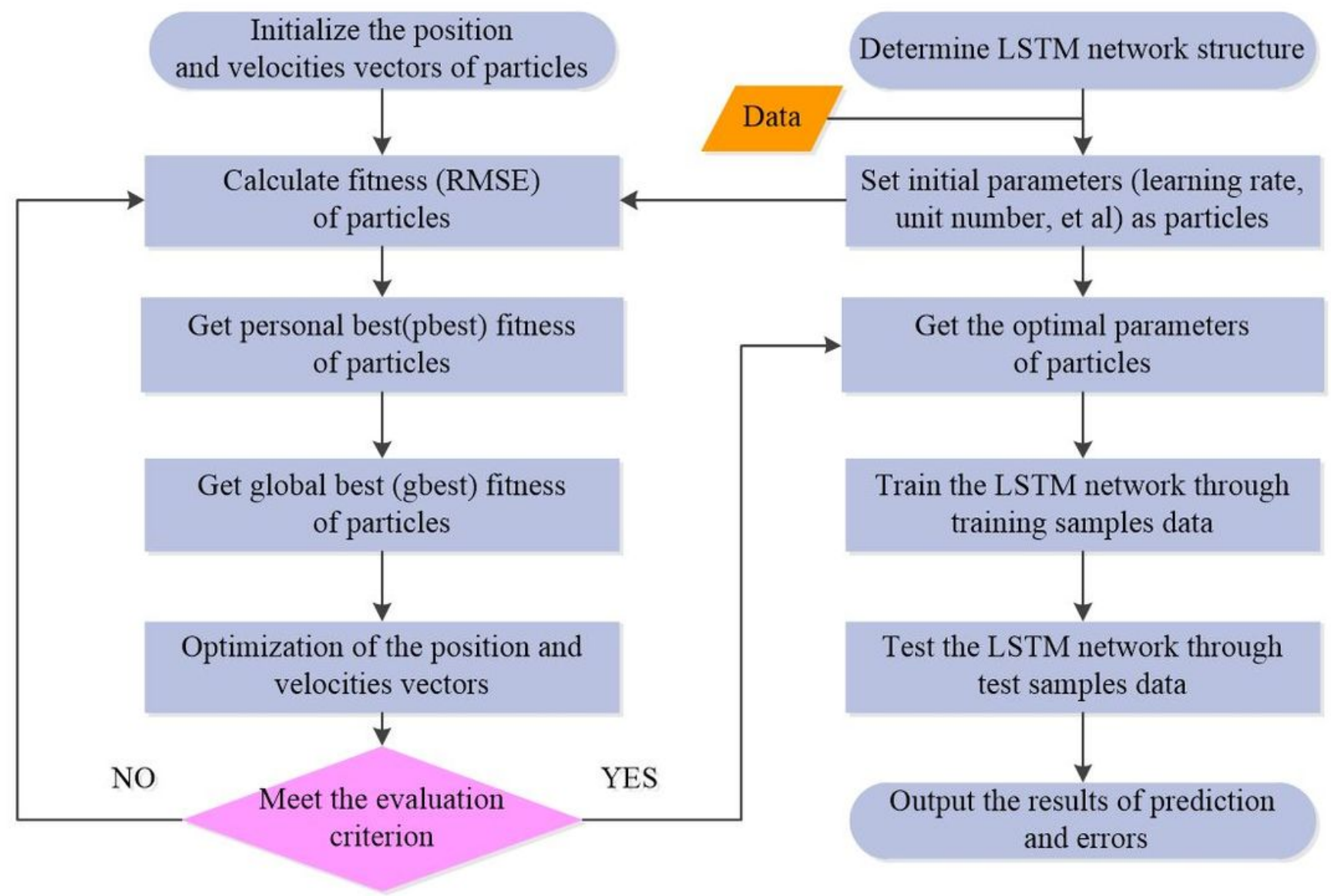

Figure 3

Flow chart of the thermal error prediction modeling process 
1st order: $\tau=1457.7 \mathrm{~s}$
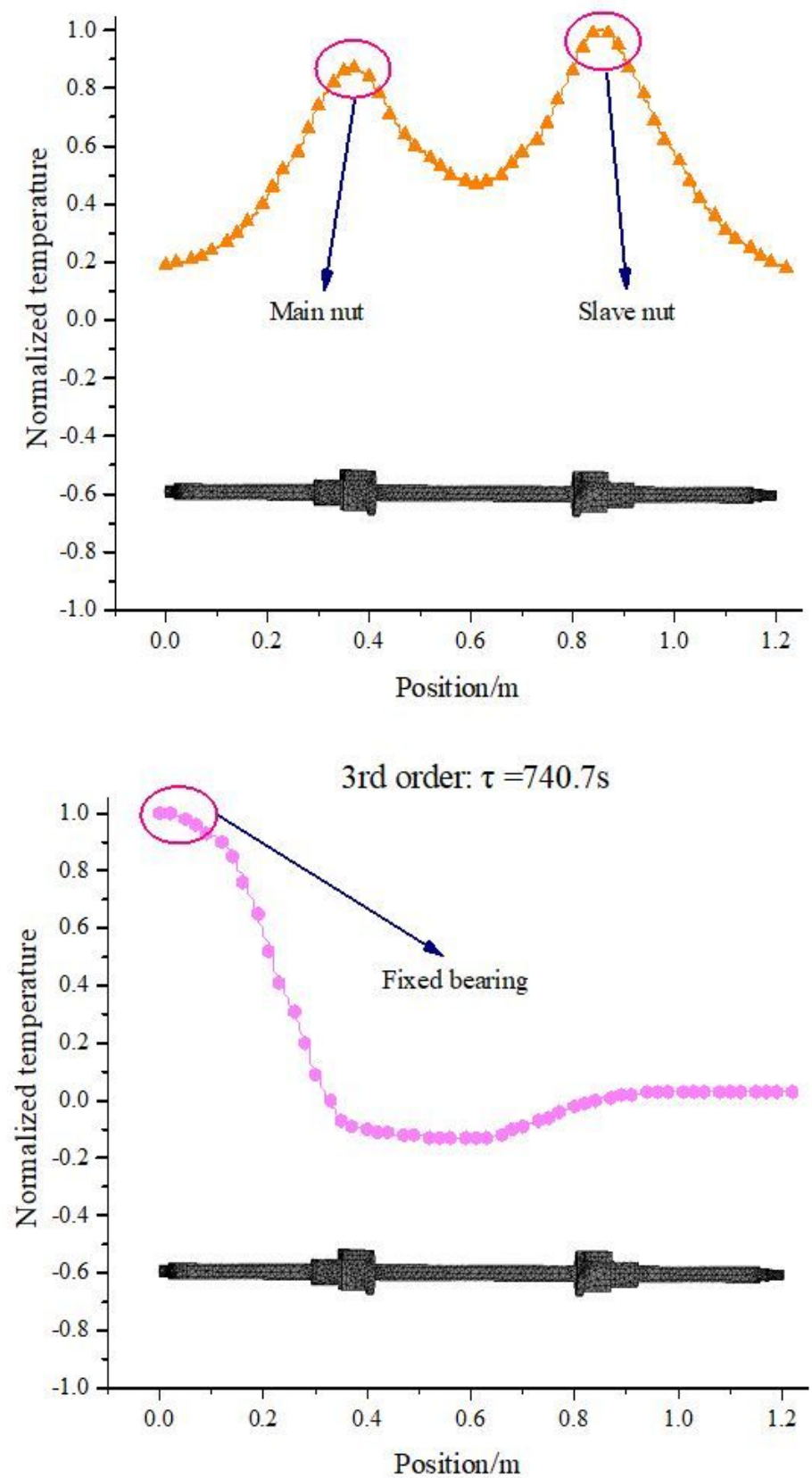
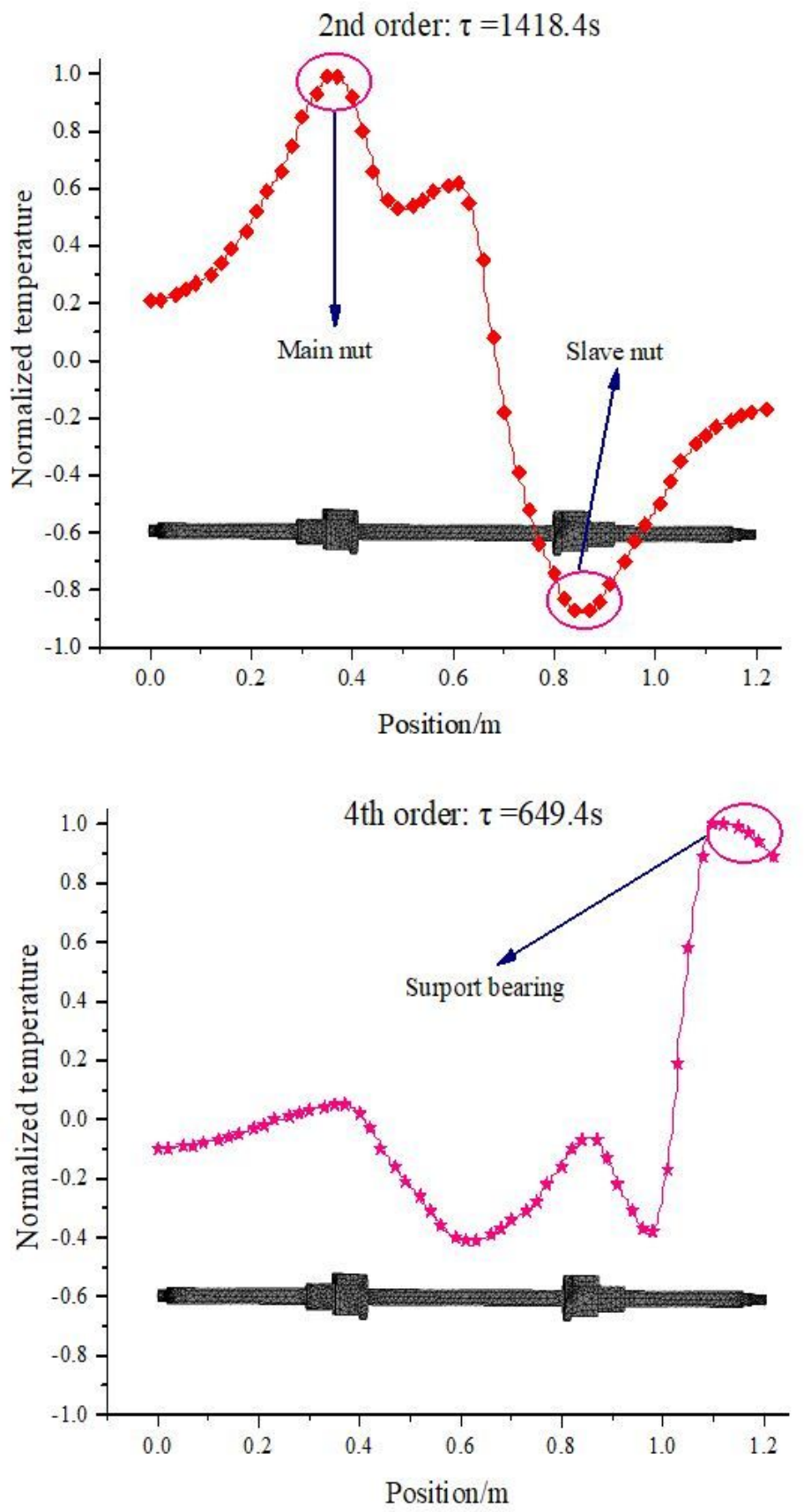

\section{Figure 4}

First four thermal modes with temperature fields and time constants 


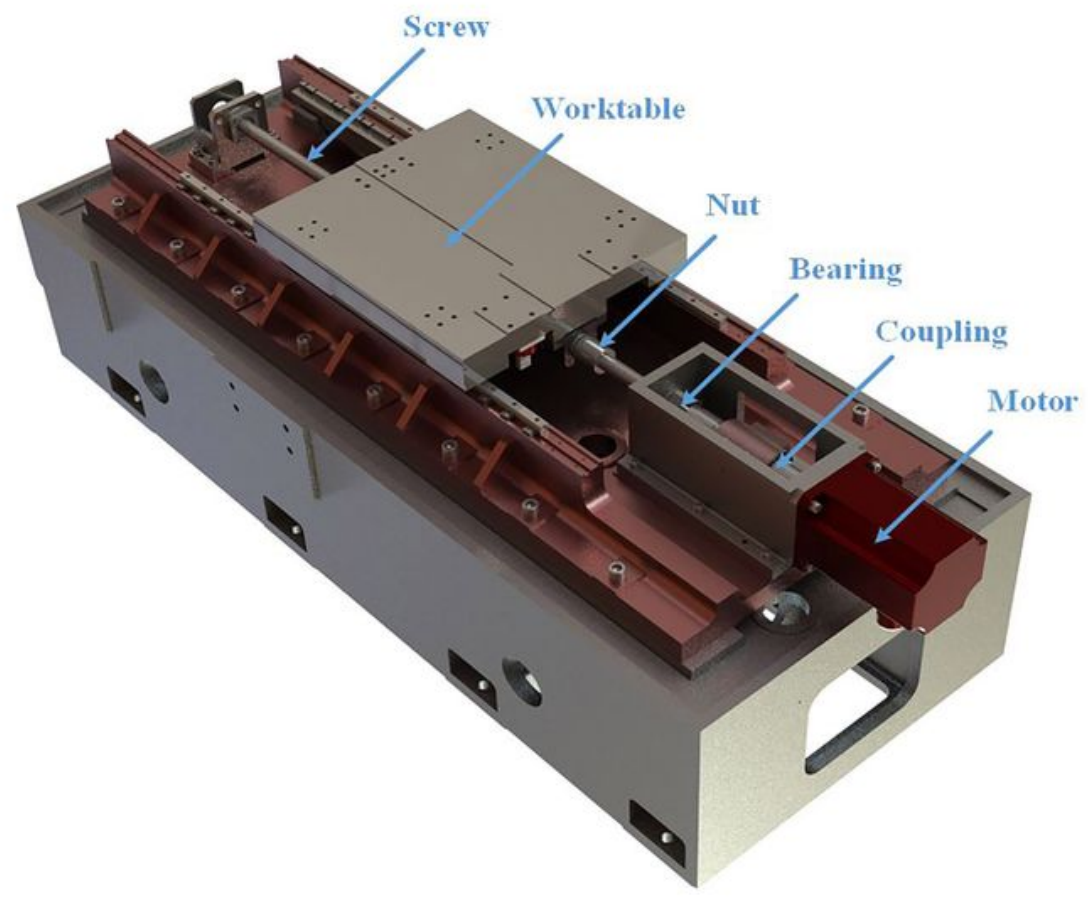

(a) Test bench of ball screws

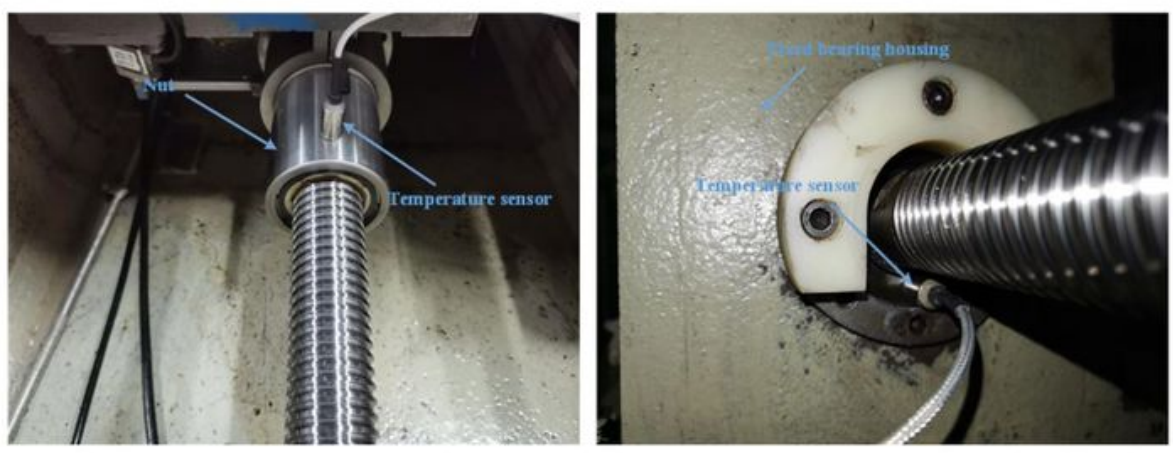

$\begin{array}{lll}\text { (b) Temperature sensor mounted on nut } & \text { (c) Temperature sensor mounted near fixed bearing }\end{array}$

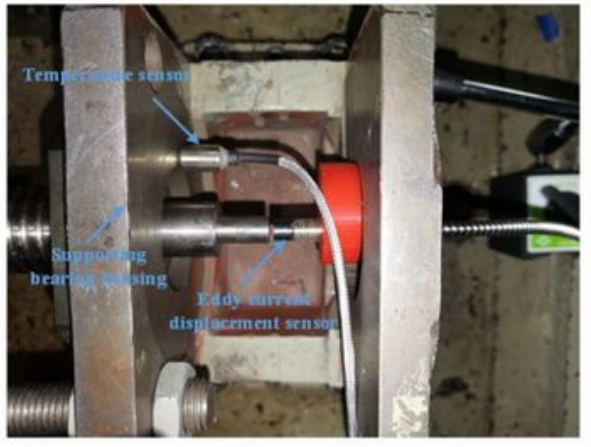

(d) Temperature and thermal deformation test

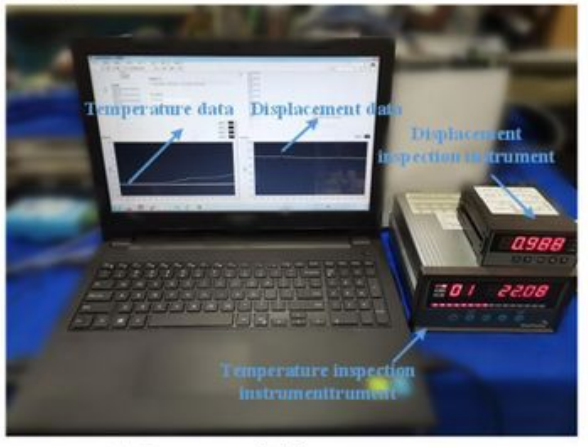

(e) Data acquisition system

\section{Figure 5}

\section{Experiment setup}




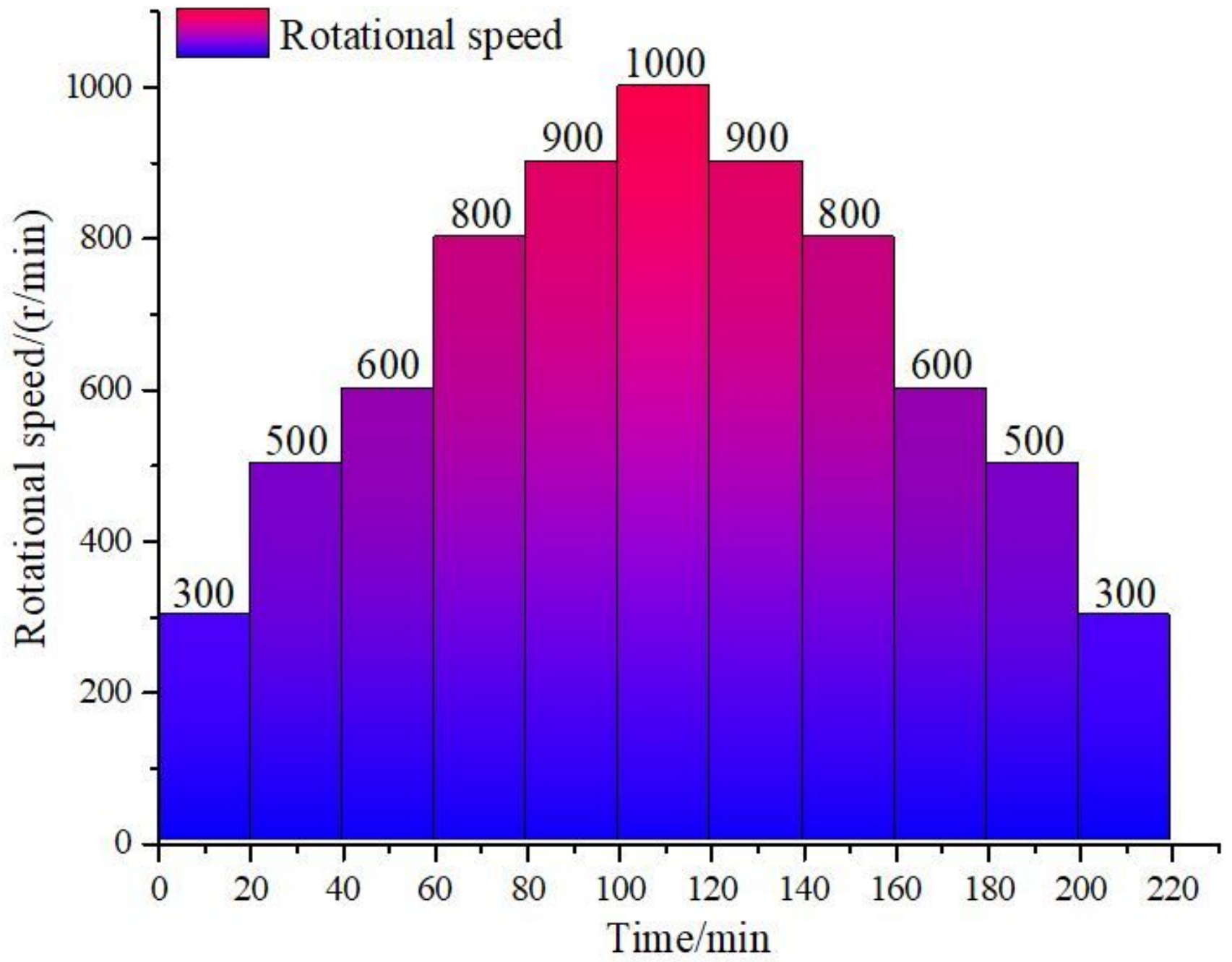

Figure 6

Step speed spectrum 


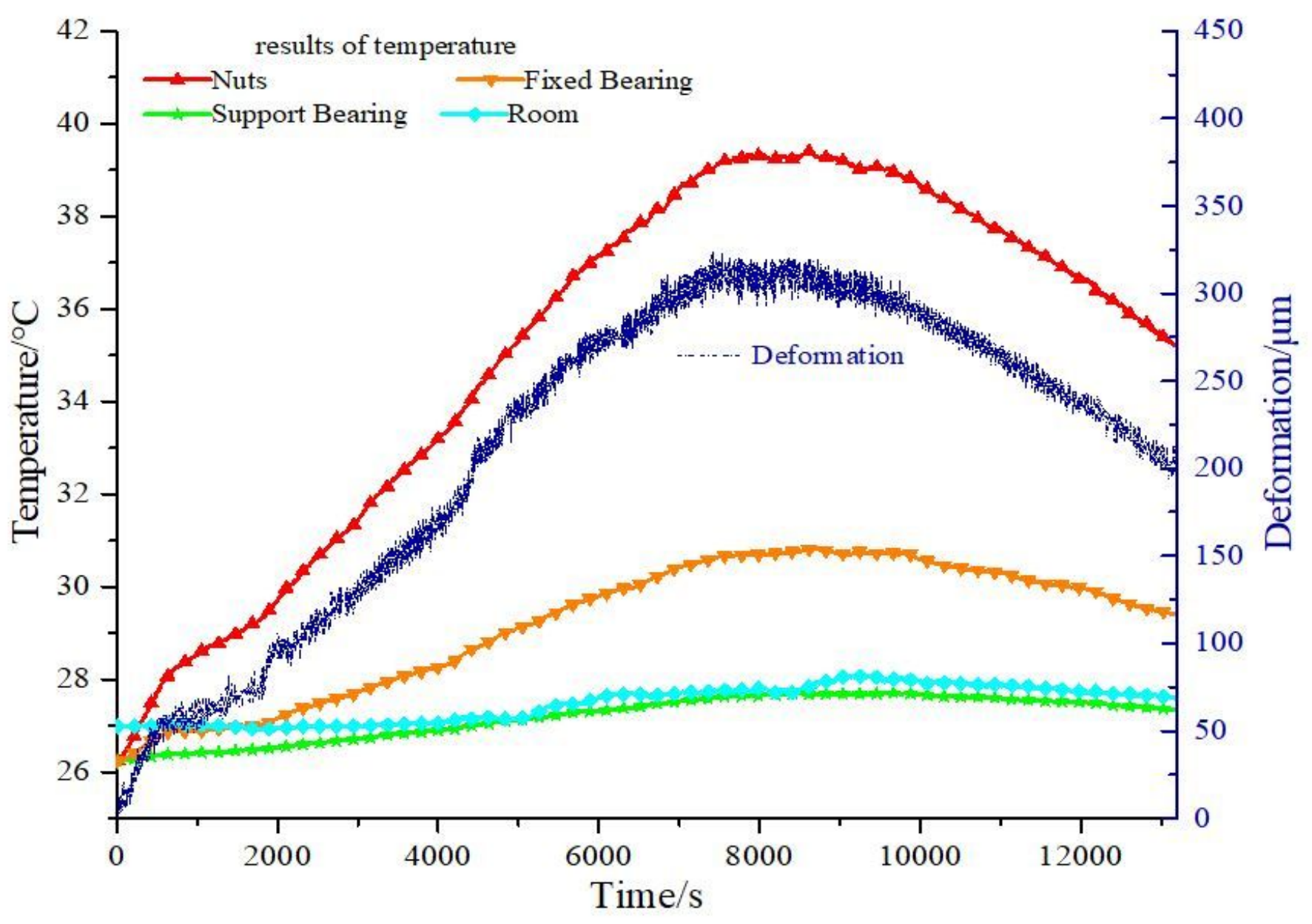

Figure 7

Experimental results of thermal error in step speed 


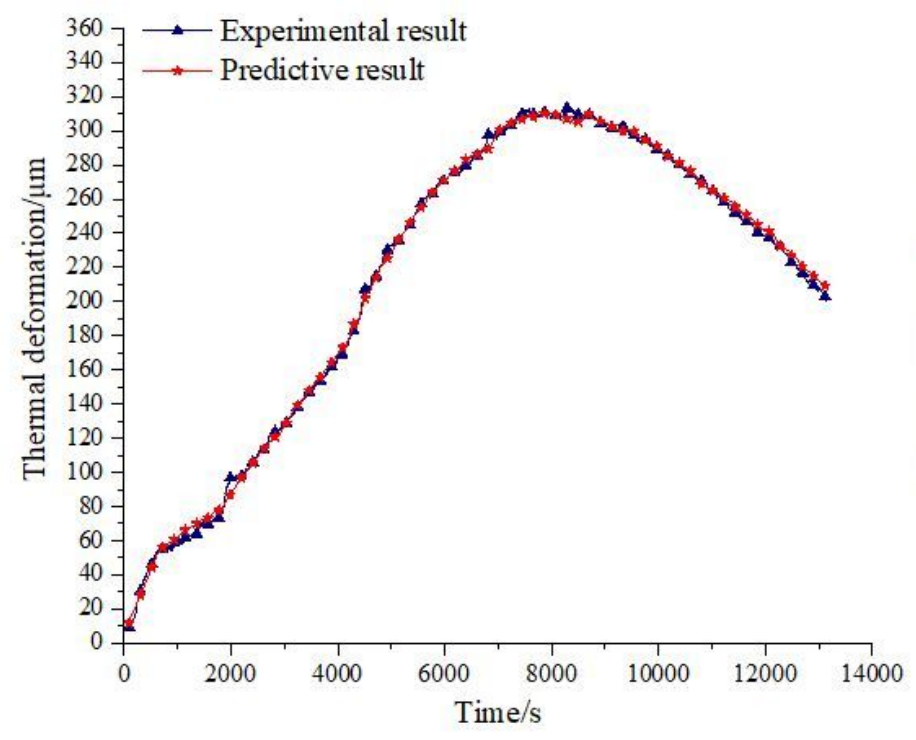

(a) Predictive results of the PSO-LSTM model

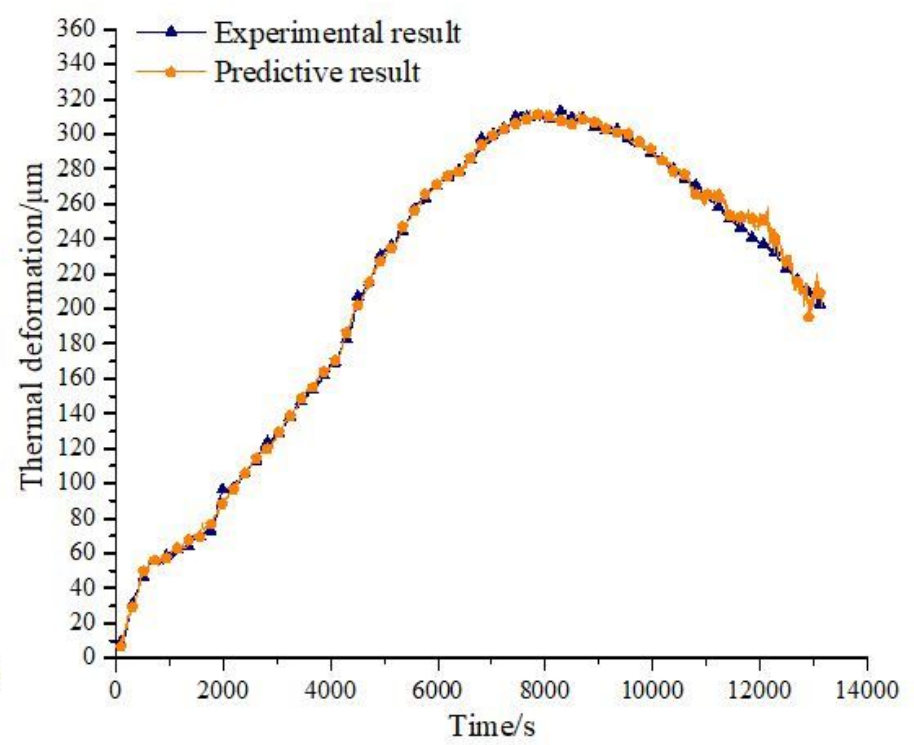

(b) Predictive results of the RBF model

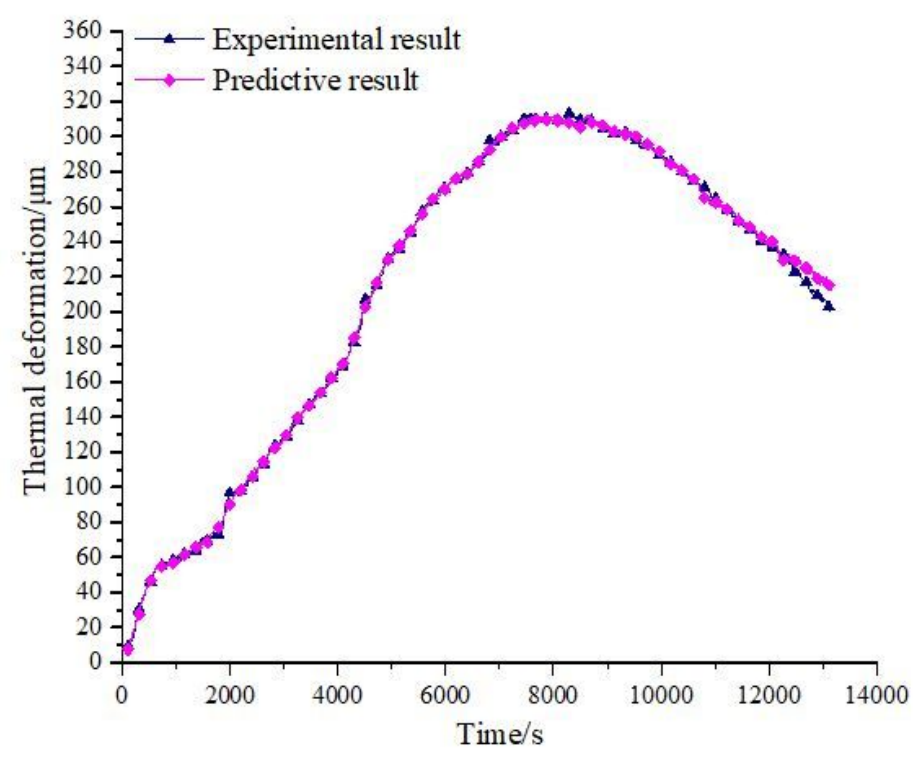

(c) The predictive results of the BP model

\section{Figure 8}

Comparative results between the experiment and the prediction of the three models 


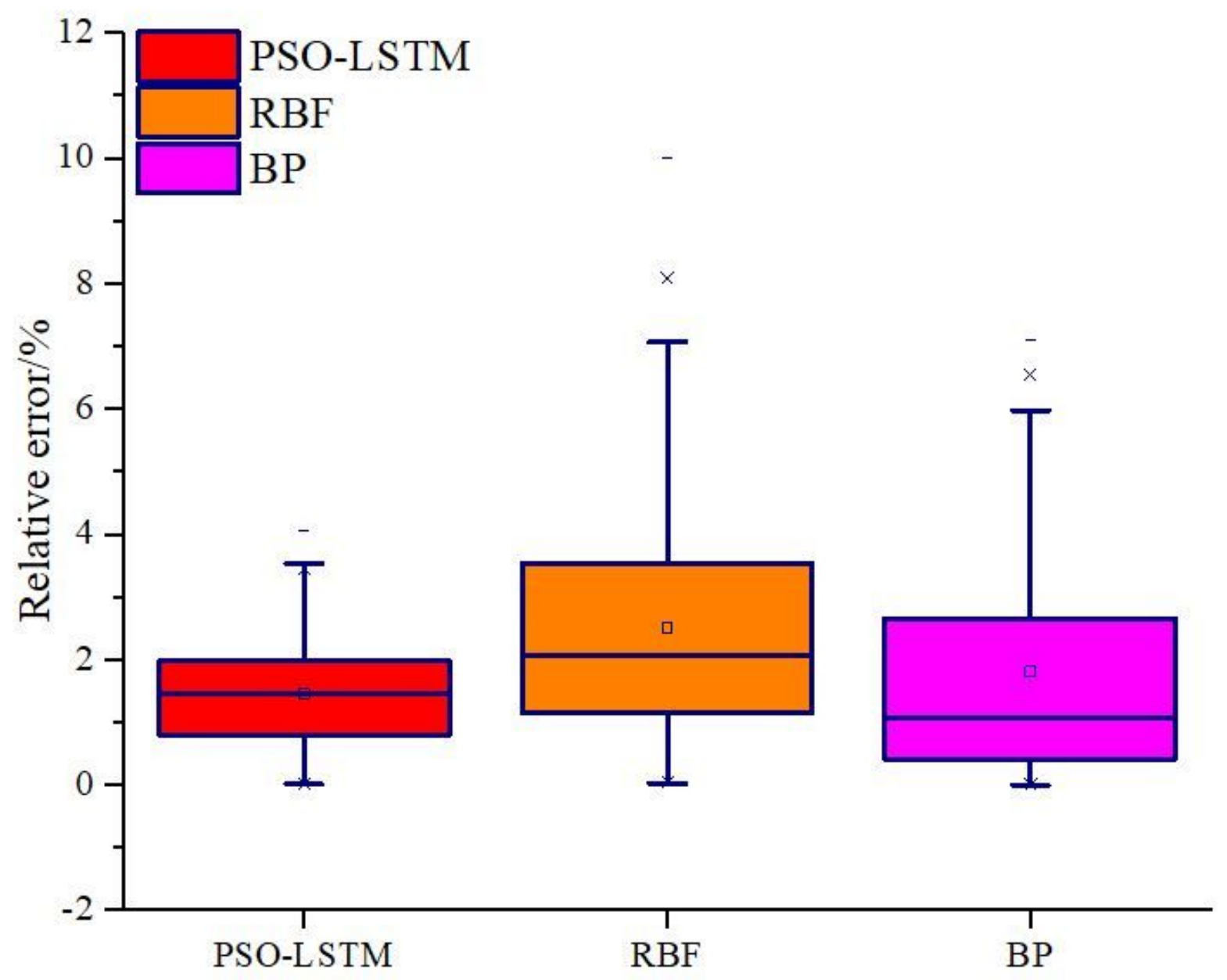

Figure 9

Absolute relative error of the three models

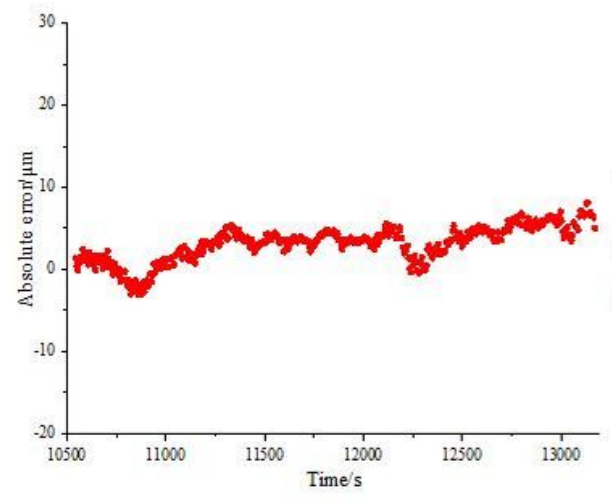

(a) PSO-LSTM model

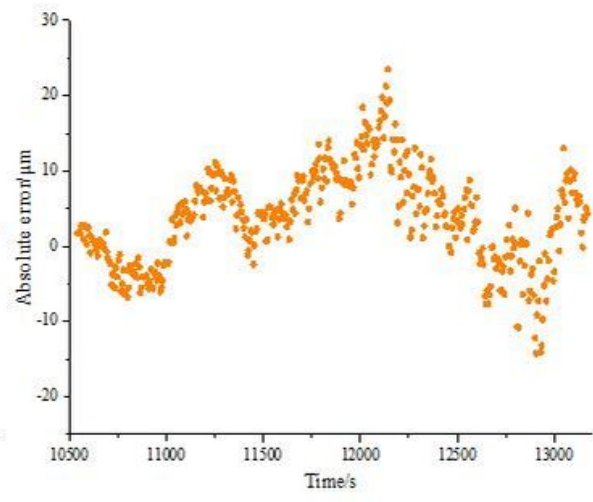

(b) RBF model

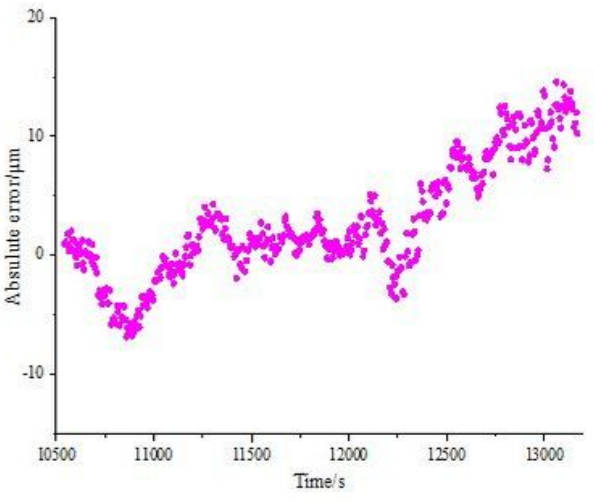

(c) BP model

Figure 10

Absolute error scatterplot of the testing set 


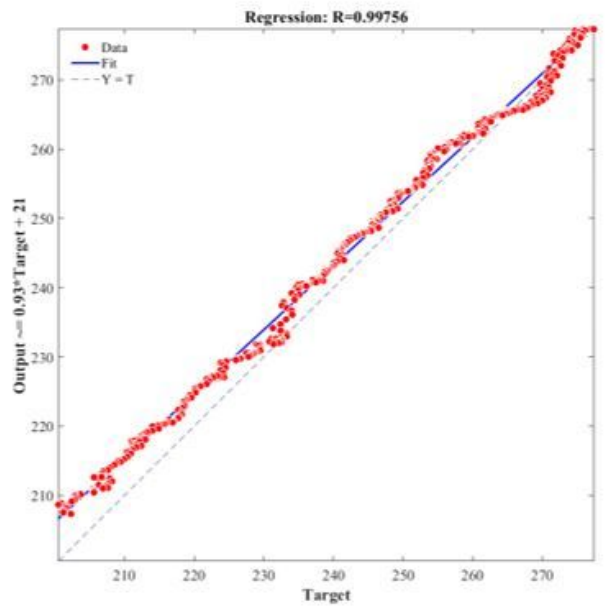

(a) PSO-LSTM model

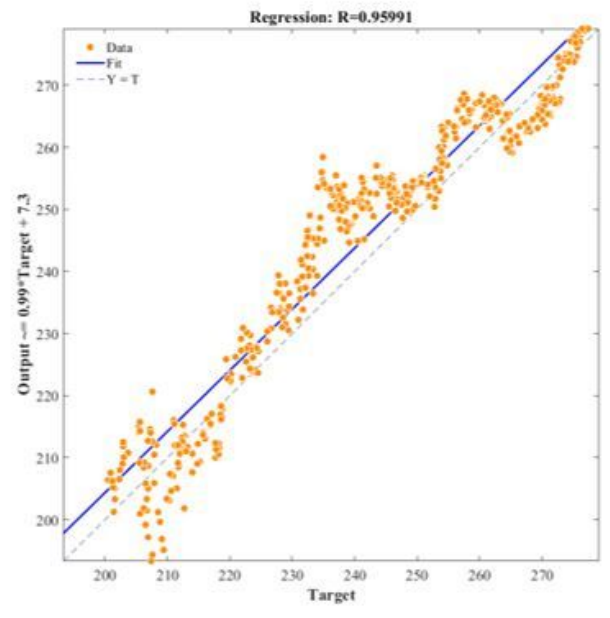

(b) RBF model

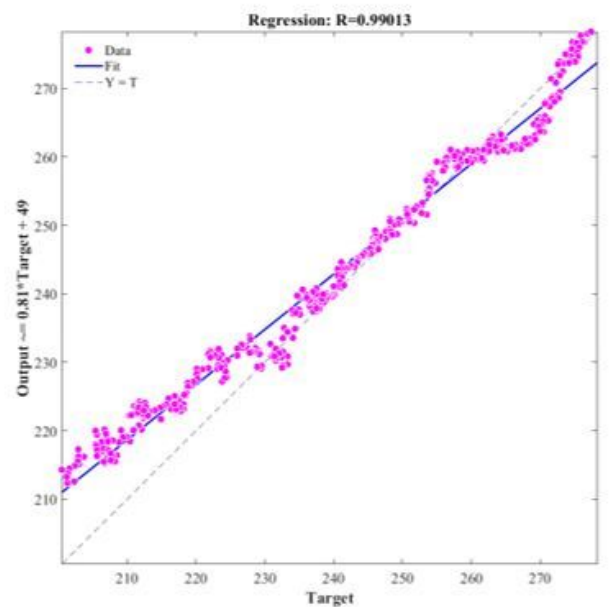

(c) BP model

Figure 11

Regression analysis

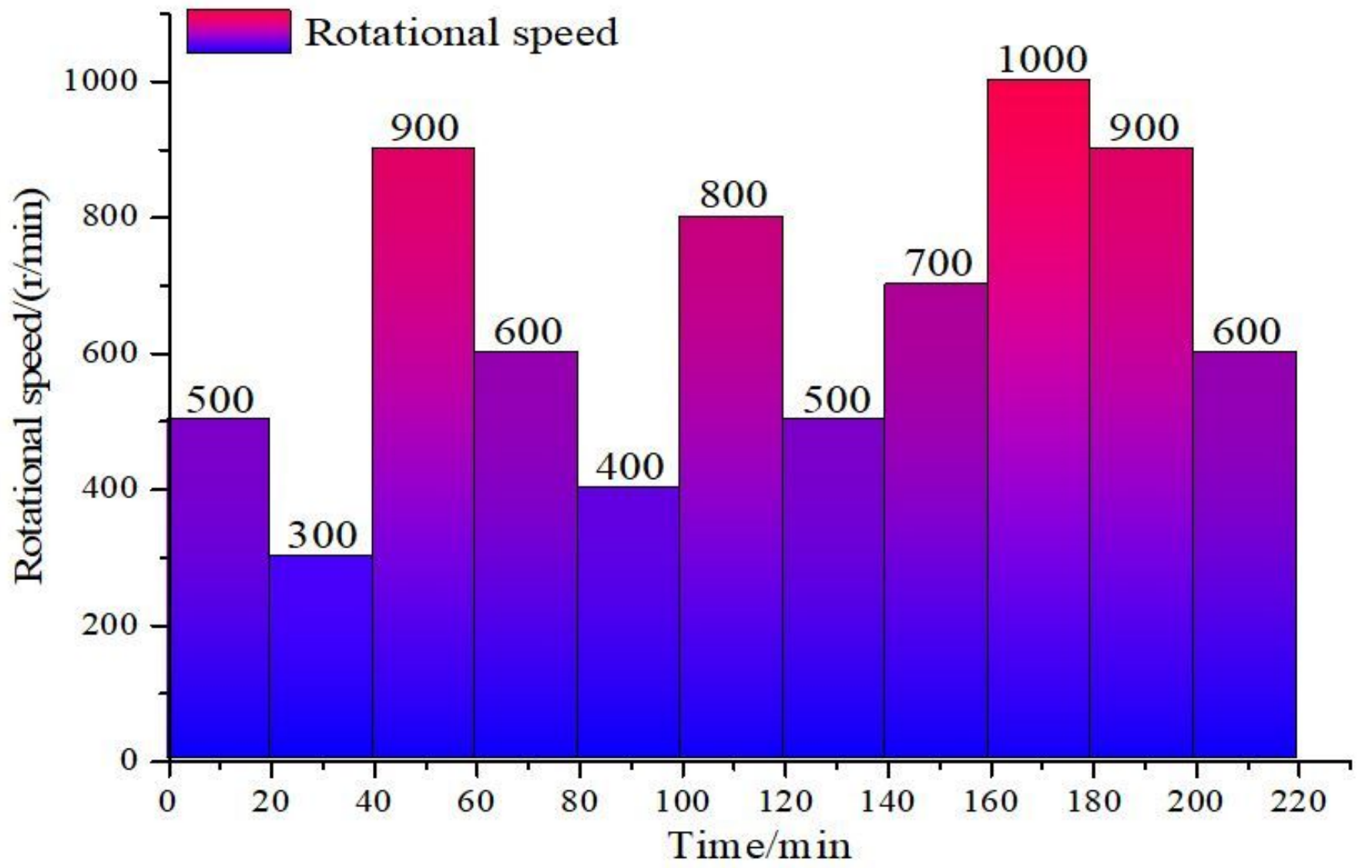

Figure 12

The random speed spectrum 


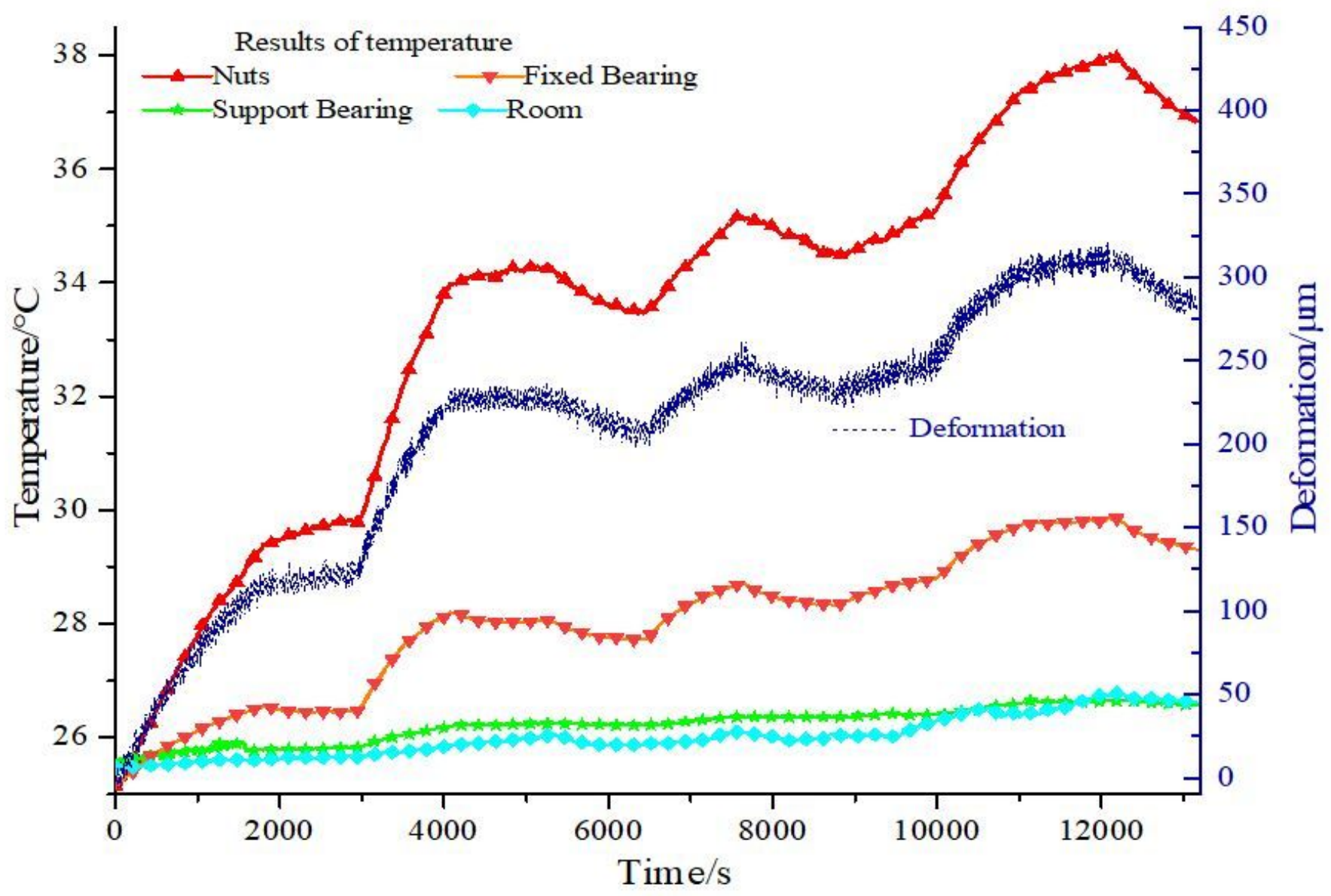

Figure 13

Experimental results of thermal error in random speed 

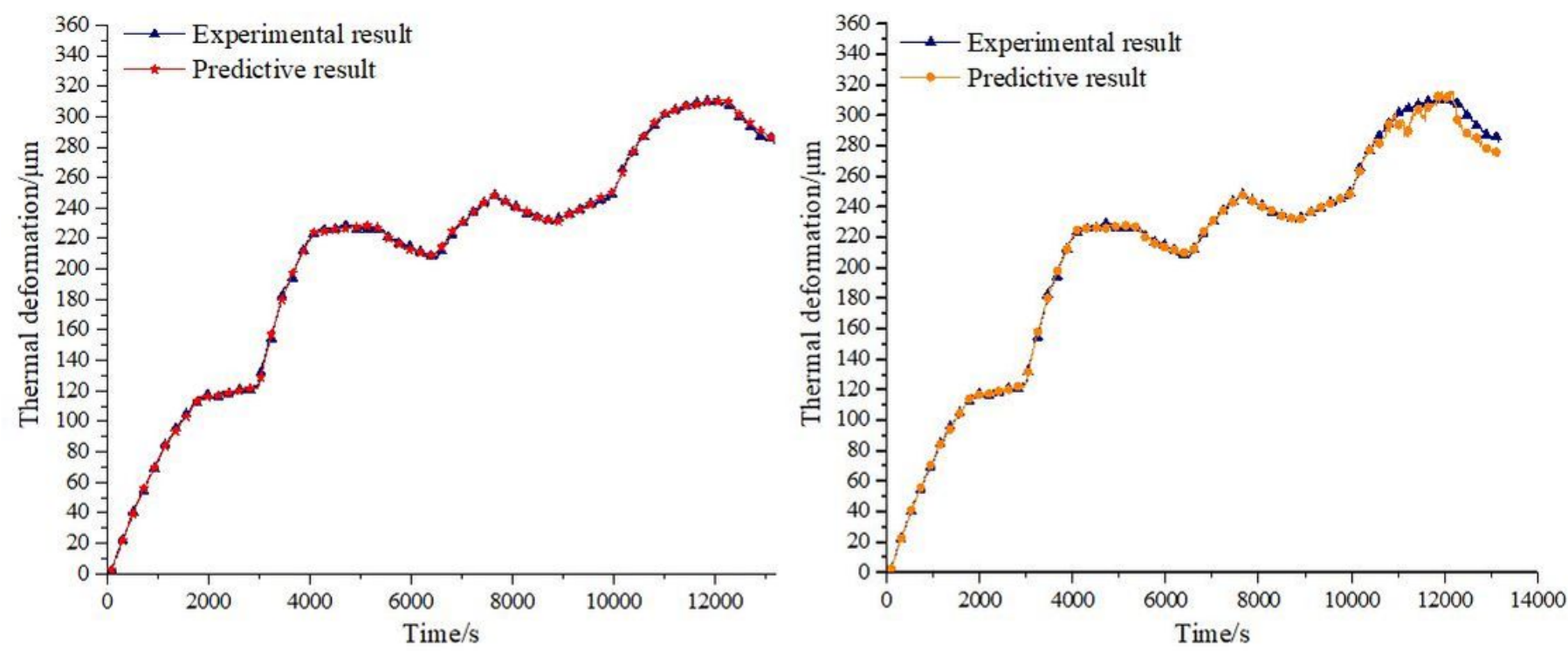

(a) The predictive results of the PSO-LSTM model (b) The predictive results of the RBF model

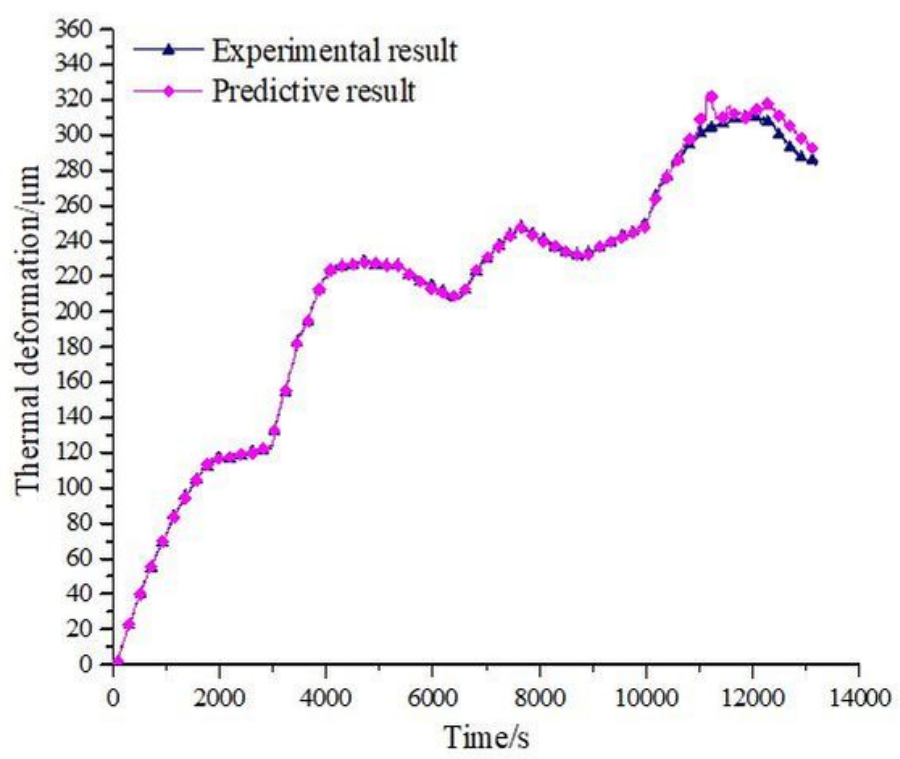

(c) The predictive results of the BP model

\section{Figure 14}

Comparative results between the experiment and the prediction of the three models. 


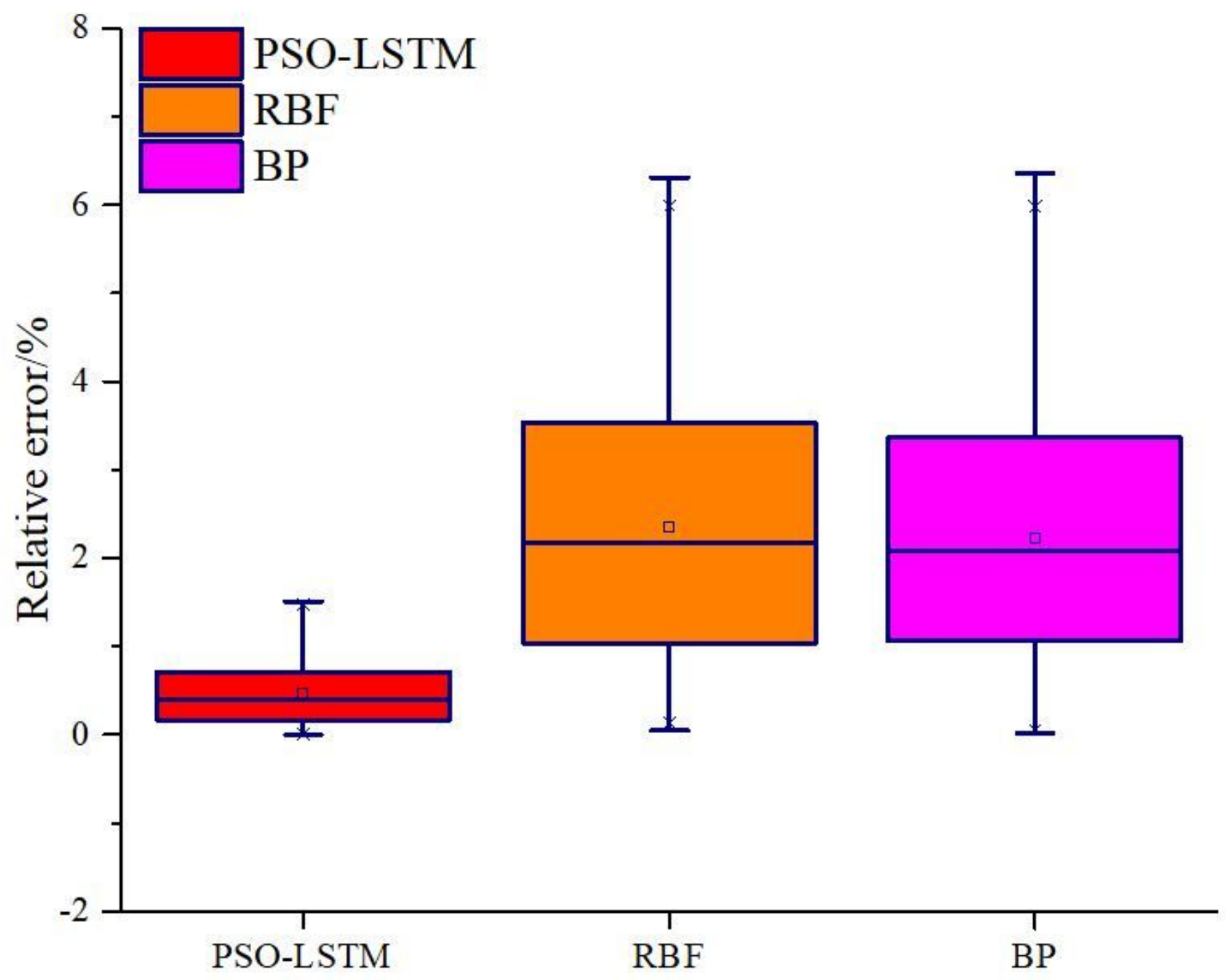

Figure 15

Absolute relative error of the three models
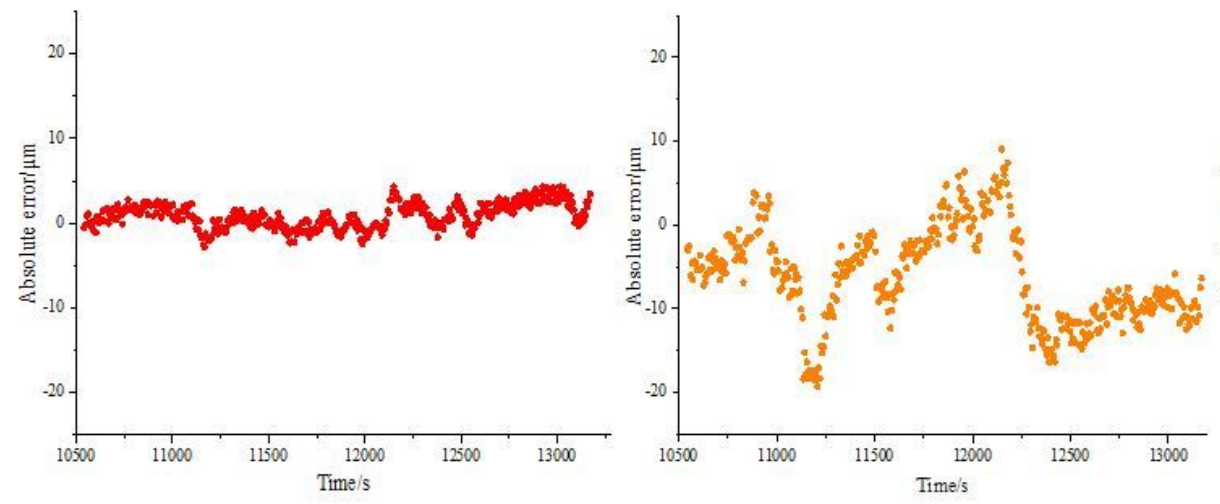

(a) PSO-LSTM model

(b) RBF model

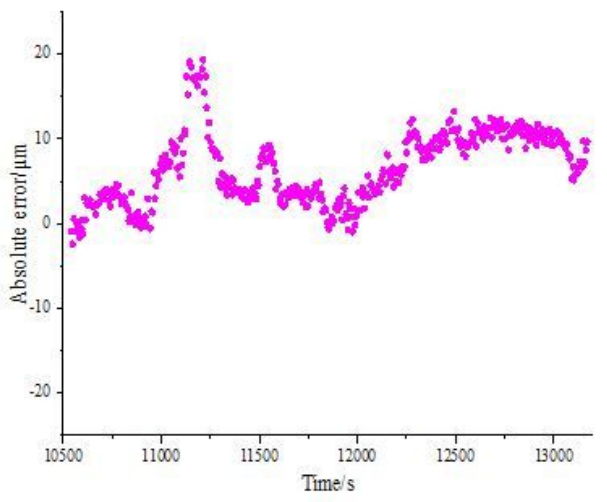

(c) BP model

Figure 16 
Absolute error scatterplot the testing set

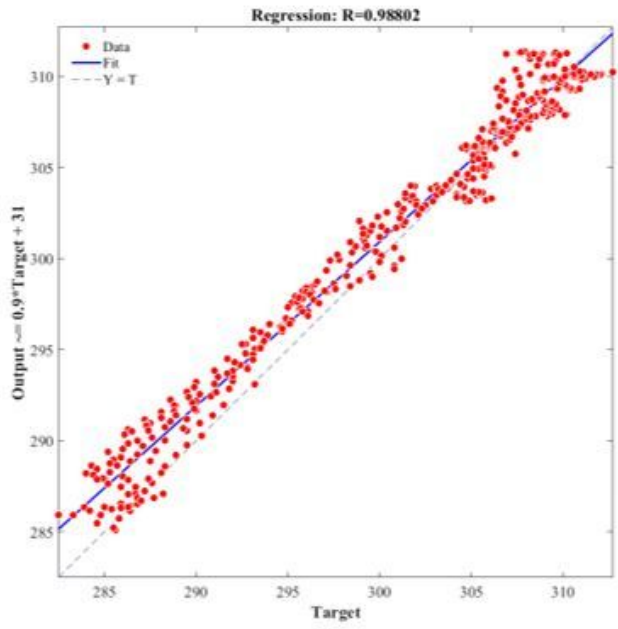

(a) PSO-LSTM model

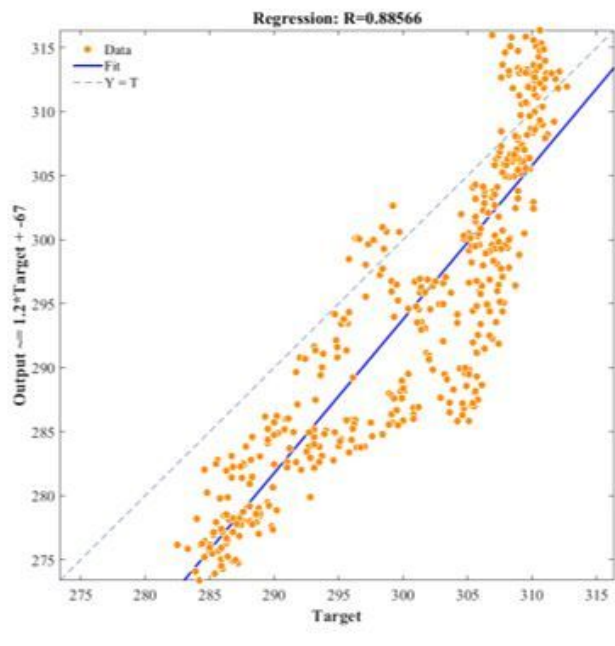

(b) RBF model

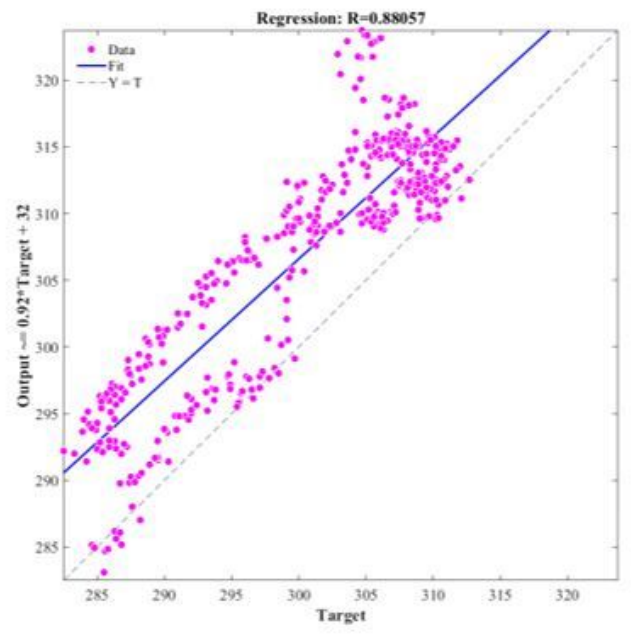

(c) BP model

Figure 17

Regression analysis curve

\section{Supplementary Files}

This is a list of supplementary files associated with this preprint. Click to download.

- Highlights.docx 